OPEN ACCESS

Edited by:

Jean Rivoal,

Université de Montréal, Canada

Reviewed by:

Raimund Tenhaken,

University of Salzburg, Austria

Claudia Vanesa Piattoni,

CONICET Santa Fe, Argentina

${ }^{*}$ Correspondence:

Leszek A. Kleczkowsk

leszek.kleczkowski@plantphys.umu.se

Specialty section:

This article was submitted to

Plant Physiology,

a section of the journal

Frontiers in Plant Science

Received: 06 July 2017 Accepted: 04 September 2017 Published: 20 September 2017

Citation:

Decker D and Kleczkowski LA (2017)

Substrate Specificity and Inhibitor Sensitivity of Plant UDP-Sugar Producing Pyrophosphorylases.

Front. Plant Sci. 8:1610. doi: 10.3389/fpls.2017.01610

\section{Substrate Specificity and Inhibitor Sensitivity of Plant UDP-Sugar Producing Pyrophosphorylases}

\author{
Daniel Decker and Leszek A. Kleczkowski* \\ Department of Plant Physiology, Umeå Plant Science Center, Umeå University, Umeå, Sweden
}

UDP-sugars are essential precursors for glycosylation reactions producing cell wall polysaccharides, sucrose, glycoproteins, glycolipids, etc. Primary mechanisms of UDP sugar formation involve the action of at least three distinct pyrophosphorylases using UTP and sugar-1-P as substrates. Here, substrate specificities of barley and Arabidopsis (two isozymes) UDP-glucose pyrophosphorylases (UGPase), Arabidopsis UDP-sugar pyrophosphorylase (USPase) and Arabidopsis UDP-N-acetyl glucosamine pyrophosphorylase2 (UAGPase2) were investigated using a range of sugar-1-phosphates and nucleoside-triphosphates as substrates. Whereas all the enzymes preferentially used UTP as nucleotide donor, they differed in their specificity for sugar-1-P. UGPases had high activity with D-Glc-1-P, but could also react with Fru-1-P and Fru-2-P ( $K_{\mathrm{m}}$ values over $10 \mathrm{mM}$ ). Contrary to an earlier report, their activity with Gal-1-P was extremely low. USPase reacted with a range of sugar-1phosphates, including D-Glc-1-P, D-Gal-1-P, D-GalA-1-P ( $K_{m}$ of $\left.1.3 \mathrm{mM}\right), \beta$-L-Ara-1-P and $\alpha$-D-Fuc-1-P $\left(K_{m}\right.$ of $\left.3.4 \mathrm{mM}\right)$, but not $\beta$-L-Fuc-1-P. In contrast, UAGPase2 reacted only with D-GlcNAc-1-P, D-GalNAc-1-P ( $K_{m}$ of $\left.1 \mathrm{mM}\right)$ and, to some extent, D-Glc-1-P $\left(K_{m}\right.$ of $\left.3.2 \mathrm{mM}\right)$. Generally, different conformations/substituents at C2, C4, and C5 of the pyranose ring of a sugar were crucial determinants of substrate specificity of a given pyrophosphorylase. Homology models of UDP-sugar binding to UGPase, USPase and UAGPase2 revealed more common amino acids for UDP binding than for sugar binding, reflecting differences in substrate specificity of these proteins. UAGPase2 was inhibited by a salicylate derivative that was earlier shown to affect UGPase and USPase activities, consistent with a common structural architecture of the three pyrophosphorylases. The results are discussed with respect to the role of the pyrophosphorylases in sugar activation for glycosylated end-products.

\footnotetext{
Keywords: enzyme structure-function analyses, enzyme substrate specificity, nucleotide sugar synthesis, UDP-fructose, UDP-fucose, UDP- $\mathrm{N}$-acetyl glucosamine pyrophosphorylase, UDP-sugar pyrophosphorylase, UDP-glucose pyrophosphorylase
}

\footnotetext{
Abbreviations: aa, amino acids; AGPase, ADP-Glc pyrophosphorylase; Ara, arabinose; Fru, fructose; Fuc, fucose; Gal, galactose; GalA, galacturonic acid; GalNAc, $N$-acetylgalactosamine; Glc, glucose; GlcA, glucuronic acid; GlcN, glucosamine; GlcNAc, $N$-acetylglucosamine; Man, mannose; NTP, nucleoside triphosphate; QSAR, quantitative structure-activity relationship; Qui, quinovose; SuSy, sucrose synthase; UAGPase, UDP-GlcNAc pyrophosphorylase; UGPase, UDP-Glc pyrophosphorylase; USPase, UDP-sugar pyrophosphorylase;; Xyl, xylose.
} 


\section{INTRODUCTION}

UDP-sugar formation is an essential pre-requirement for any cell to produce larger and more complex carbohydrate-containing compounds. Production of UDP-sugars involves either primary mechanisms, where an UDP-sugar is produced from a sugar molecule that is activated by linking it with an UDP moiety, resulting in a more reactive compound (Kleczkowski and Decker, 2015), or secondary mechanisms, where an already produced UDP-sugar is converted to another one (Reiter, 2008; Yin et al., 2011). In plants, the primary mechanisms of UDP-sugar formation involve three UTP-dependent pyrophosphorylases, differing in specificity for sugar-1-P, which serves as second substrate. UDP-glucose pyrophosphorylase (UGPase) is considered to be more or less specific for glucose-1-P (Glc-1-P), and has by far the highest activity among the pyrophosphorylases (Kleczkowski et al., 2010; Kleczkowski and Decker, 2015). This is in contrast to UDP-sugar pyrophosphorylase (USPase), which uses a variety of sugar-1-phosphates as substrates (Kotake et al., 2004; Damerow et al., 2010; Kleczkowski et al., 2011a), and to UDP- $N$-acetylglucosamine pyrophosphorylase (UAGPase), which prefers $N$-acetylglucosamine-1-P (GlcNAc-1-P) and $\mathrm{N}$-acetylgalactosamine-1-P (GalNAc-1-P) as substrates (Yang et al., 2010). All these enzymes are predominantly located in the cytosol (Kleczkowski et al., 2010; Kleczkowski and Decker, 2015), with the exception of a unique type of UGPase, which is entirely based in chloroplasts where it serves as an essential step in sulfolipid biosynthesis (Okazaki et al., 2009). As the synthesis of the UDP-sugars is a fully reversible reaction, each pyrophosphorylase can also be involved in the production of a given sugar-1-P from the respective UDP-sugar, and thus contributing to an equilibrium concentration between those metabolites.

Once made by the pyrophosphorylases, UDP-sugars then serve as substrates to a myriad of glycosyltransferase activities, which transfer the monosaccharide residue from a given UDP-sugar to an acceptor molecule. The acceptor could be another sugar (e.g., for sucrose or trehalose formation), a polysaccharide (e.g., cellulose, hemicellulose, pectin formation), a protein (glycoprotein formation), a lipid (glycolipid formation), and many others (Feingold and Avigad, 1980; Lairson et al., 2008; Yonekura-Sakakibara, 2009; Bar-Peled and O’Neill, 2011). A given UDP-sugar molecule can also be interconverted to another UDP-sugar by the action of specific epimerases and via other mechanisms, including - among others a conversion of UDP-Glc to UDP-glucuronic acid (UDPGlcA) by UDP-Glc dehydrogenase (Kleczkowski and Decker, 2015).

Extracts from higher plants have been shown to contain about 30 different types of nucleotide sugars, most of them UDP-sugars, and for some of them no known synthetic pathways have been demonstrated (Bar-Peled and O'Neill, 2011). Given the metabolic importance of UDP-sugars and the fact that they serve as precursors to most of biomass in nature (Kotake et al., 2010), surprisingly little is known about substrate specificity of the pyrophosphorylases catalyzing the primary reactions leading to glycosylated end-products, and even less about the relative contribution of each enzyme to these cellular pathways.

To contribute to that, in the present study, we measured the activities of several purified recombinant UDP-sugar producing pyrophosphorylases and compared their substrate specificities with respect to an array of sugar-1-P. The structure/function analyses of sugar-1-phosphates and their binding sites in the homology-derived structural models for the three pyrophosphorylases were compared with the substrate specificities that were experimentally determined for these enzymes. We also demonstrated that an inhibitor earlier shown to affect UGPase and USPase activities (Decker et al., 2017), had similar effects on purified UAGPase2. The data are discussed with respect to structural determinants of substrate binding and to possible roles of each of the pyrophosphorylases in vivo.

\section{MATERIALS AND METHODS}

\section{Recombinant UGPases, USPase and UAGPase2}

Barley UGPase, Arabidopsis UGPase1 and UGPase2, and Arabidopsis USPase were heterologously expressed in Escherichia coli and purified to homogeneity as earlier reported (Martz et al., 2002; Meng et al., 2008; Decker et al., 2014, 2017). An expression construct containing cDNA of Arabidopsis UAGPase2 (At2g35020) was order-made by GenScript, San Francisco, CA, United States. The construct contained a nucleotide sequence that was optimized for bacterial expression. The full coding sequence was cloned into prokaryotic expression vector pET22b + (Novagen) in fusion with a poly-His affinity tag (at $\mathrm{C}$ terminus). The construct was sequenced on both strands using a primer walking strategy with unlabeled primers (Cybergene, Huddinge, Sweden) and BigDye Terminator Cycle sequencing kit (Perkin Elmer), and transformed into BL21 (DE3) E. coli cells. The His 6 -tagged UAGPase2 was overexpressed and purified in the Protein Expertise Platform (Chemistry Department, Umeå University), using immobilized metal $\left(\mathrm{Ni}^{2+}\right)$ affinity chromatography. The purified UAGPase2 was subsequently snap-frozen in liquid nitrogen for storage. Details of bacteria transformation were as earlier described for plant UGPase (Meng et al., 2009a).

In Supplementary Table S1, we have summarized details of preparation, expression and purification of recombinant enzymes used in this study, along with relevant details from other studies on the same enzymes.

\section{Assays}

The activities of UGPases, USPase and UAGPase2 were determined in the forward direction of their reactions, using an assay based on quantification of the Pi released from inorganic pyrophosphate $(\mathrm{PPi})$, the product of the pyrophosphorylase reaction. Procedures followed were generally those described in Litterer et al. (2006a) and Decker et al. (2012). Assays (each in a final volume of $50 \mu \mathrm{l}$ ) were run on 96-well plates (Sarstedt, Germany) and contained $100 \mathrm{mM}$ Hepes (pH 7.5), $5 \mathrm{mM} \mathrm{MgCl}$, 0.5 unit of inorganic pyrophosphatase (Roche, Switzerland), an 
aliquot of a purified UGPase, USPase or UAGPase2 and varied concentrations of a sugar-1-P and a NTP (detailed concentrations are given in Figure legends). Reactions were initiated by addition of a pyrophosphorylase, were run at room temperature for $12 \mathrm{~min}$, and were terminated by addition of $50 \mu \mathrm{l} \mathrm{Pi-detection}$ solution (for final concentration: $100 \mathrm{mM}$ acetate, $0.7 \%$ ascorbic acid and $1.5 \%$ ammonium molybdate). Reactions were developed at room temperature for $5 \mathrm{~min}$ and the absorbance at $720 \mathrm{~nm}$ was measured to determine the amount of the blue colored phosphate-molybdenum complex that was proportional to the amount of phosphate present. The amount of produced Pi was quantified with a Pi standard curve.

Effects of different inhibitors on UGPase, USPase and UAGPase activities were assayed in the direction of Glc-1-P and UTP formation, using a coupling enzyme system, as described in Decker et al. (2012). The assays (300 $\mu \mathrm{l}$ each) contained $100 \mathrm{mM}$ Hepes (pH 7.5), $5 \mathrm{mM} \mathrm{MgCl}_{2}, 0.3 \mathrm{mM}$ NADP+, 0.5 unit Glc-6-P dehydrogenase (Roche), 0.5 unit phosphoglucomutase (Sigma Aldrich), and concentrations of UDP-Glc and PPi at their $K_{\mathrm{m}}$ values for a given enzyme: for UGPase - 0.034 and $0.039 \mathrm{mM}$, respectively (Meng et al., 2009a); for USPase -0.3 and $0.16 \mathrm{mM}$, respectively (Litterer et al., 2006b); and for UAGPase2 0.21 and $0.32 \mathrm{mM}$, respectively, (see Supplementary Figure S5). Assays were started by addition of a pyrophosphorylase, and were carried out by monitoring Glc-1-P production via its coupling to the formation of NADPH at $340 \mathrm{~nm}$ using Beckman DU 530 spectrophotometer.

In all activity determinations, assays were done at least twice for each experimental point, and the variation was usually less than $10 \%$. A unit of enzymatic activity is defined as amount of the enzyme required either to produce $1 \mu \mathrm{mol}$ of PPi per min (forward reaction) or to produce $1 \mu \mathrm{mol}$ of Glc-1-P per min (reverse reaction).

\section{QSAR Analyses}

In order to extend the range of possible sugar-1-phosphates as substrates of the pyrophosphorylases, we used the QSAR approach. The QSAR was based on Free-Wilson model (Free and Wilson, 1964), which analyzes properties of compounds that were experimentally verified to be active in a given system, and provides a list of related compounds, which are likely to be active. Since the pyrophosphorylase reactions are freely reversible (Kleczkowski and Decker, 2015), for QSAR modeling we used chemical structures of nucleotide-sugars rather than corresponding active sugar-1-phosphates. These nucleotidesugars were classified using so called 1D descriptors, such as the configuration of hydroxyl groups on C1, C3, C4 of a sugar moiety, nucleotide-type and substituents/configurations on C2 and C5 of a sugar. The contribution to the biological activity of each descriptor was estimated using Excel Solvers non-linear GRG (Microsoft, Redmond, WA, United States).

\section{Homology Modeling and Analyses of Substrate Binding}

For analyses of plant UGPase substrate binding interactions, we used crystal structure of Arabidopsis UGPase1 with bound
UDP-Glc (PDB code 2ICY) (McCoy et al., 2007). Homology models of Arabidopsis USPase and Arabidopsis UAGPase2 were constructed using crystal structures of Leishmania major USPase (with UDP-Glc bound) (PDB code 3OH4) (Dickmanns et al., 2011) and human UAGPase2 (with UDP-GlcNAc bound) (PDB code 1JV1) (Peneff et al., 2001), respectively. Modeled structures were obtained using SWISS-model (Biasini et al., 2014), and the final accepted models had a GMQE (a quality estimation which combines properties from the target-template alignment) of 0.67 and 0.71 for USPase and UAGPase2, respectively. The comparison of the active sites of UGPase vs. USPase and UAGPase 2 was performed using LigPlot+ (Laskowski and Swindells, 2011).

\section{Chemicals}

All sugar-1-phosphates, except D-Glc-1-P, fructose-phosphates and $\alpha$-D-glucosamine-1-P [GlcN-1-P], were kindly provided by Dr. Motomitsu Kitaoka, from Laboratory of Enzyme Research, NARO, Ibaraki, Japan. Separate batches of Gal-1-P were also purchased from Sigma-Aldrich (Stockholm, Sweden) and from Carbosynth (Compton, United Kingdom). Glc-1-P, glucosamine-1-P (GlcN-1-P) as well as $\beta$-D-fructofuranosyl1-P $\quad(\beta$-D-Fruf-1-P), $\beta$-D-fructofuranosyl-2-P $\quad(\beta$-D-Fruf-2-P) and other fructose-phosphates were from Sigma-Aldrich. Compound \#41 (ZINC720558), an inhibitor of Trypanosoma brucei UAGPase2 (Urbaniak et al., 2013), was purchased from MolPort (Riga, Latvia), whereas cmp \#6D (CID 6526371) was from (ChemBridge Inc., San Diego, CA, United States).

\section{RESULTS}

\section{Activities of UGPases with Different Sugar Phosphates and NTPs}

To study substrate specificity of plant UGPases, we have used purified recombinant barley UGPase as well as UGPase1 and UGPase2 from Arabidopsis. The Arabidopsis enzymes were the first UGPase isozymes which had been characterized from a single plant species, and they had remarkably similar physical and kinetic properties (Meng et al., 2008). Each of the enzymes was screened for activity against 55 substrate combinations (11 sugar-1-phosphates and 5 different NTPs). The reasons for this wide choice of potential substrates were twofold: (i) to identify/verify the mechanisms of synthesis of nucleotide sugars reported for plant extracts (Bar-Peled and O'Neill, 2011), and (ii) to rationalize a structure-activity relationship for sugar-moiety of a nucleotide sugar with respect to substrate binding sites of distinct pyrophosphorylases (see below).

The assays revealed that plant UGPases are highly specific for Glc-1-P and UTP as substrates (Figure 1), as earlier reported (Nakano et al., 1989; Kimura et al., 1992; Ritter et al., 1996; Kleczkowski et al., 2004; Meng et al., 2008). However, the three UGPases reacted also with Fru-1-P, using a variety of NTPs as a second substrate (Figure 1). These activities were in the range of 7-20\% of those seen with Glc-1-P and UTP. The Fru-1-Pdependent activity, although low, could be increased at least 2-fold upon increasing Fru-1-P from 1 to $5 \mathrm{mM}$ (data not shown), 


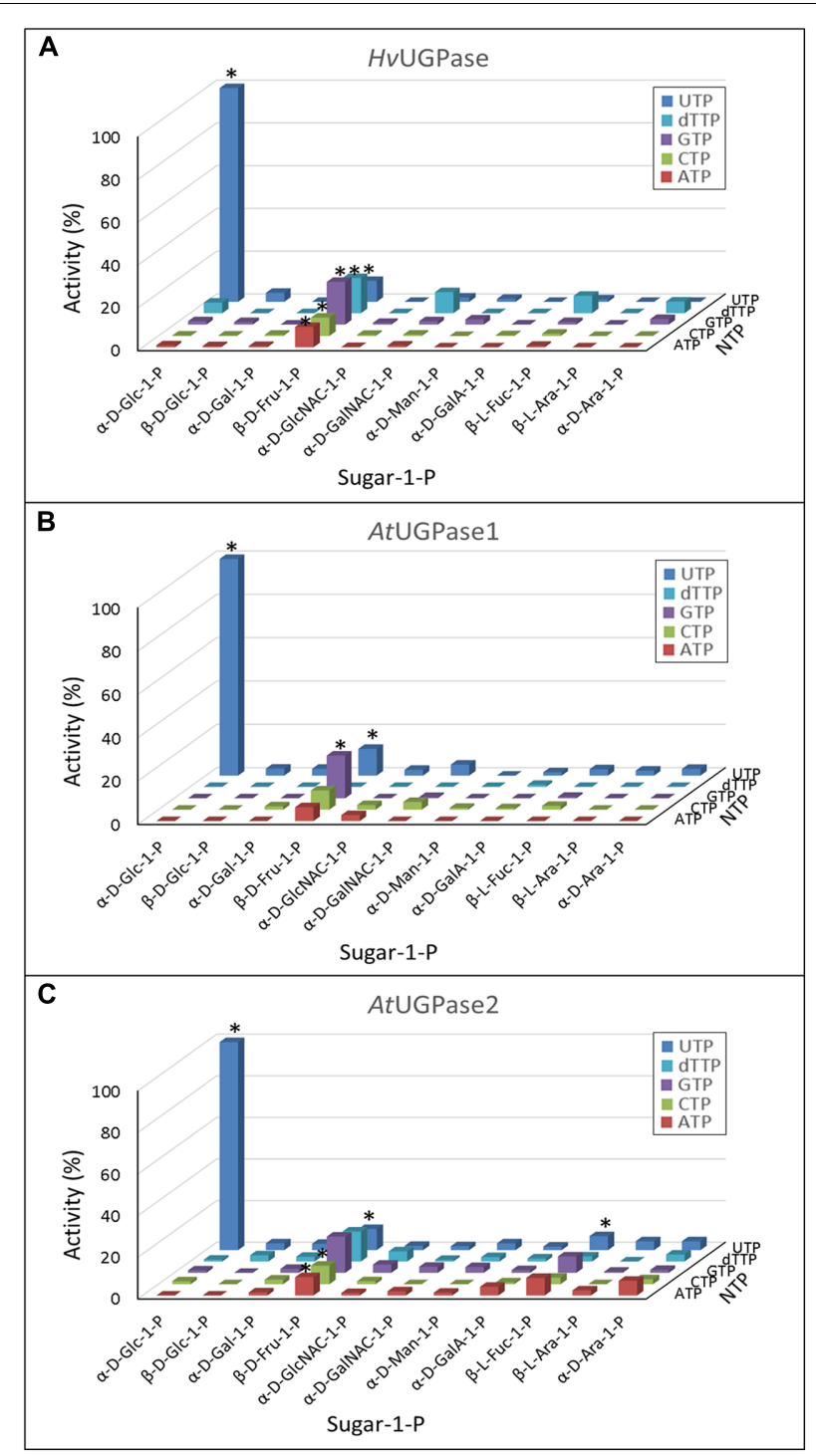

FIGURE 1 | (A-C) Substrate specificity of barley UGPase as well as Arabidopsis UGPase-1 and UGPase-2. Each assay contained a sugar-1-P (at $1 \mathrm{mM}$ ) and a nucleoside triphosphate (at $1 \mathrm{mM}$ ). $100 \%$ of activity refers to 1100 units/mg protein for barley UGPase and to 29 and 11 units/mg protein for Arabidopsis UGPase1 and UGPase2, respectively. * indicates significant (Student's test) differences from background $(n=2, p<0.05)$.

suggesting a low affinity for this substrate. Indeed, the determined $K_{\mathrm{m}}$ values of barley UGPase and Arabidopsis UGPase1 with Fru1-P were very high (Supplementary Figure S1) and difficult to determine accurately, as Fru-1-P at and above $3 \mathrm{mM}$ caused clouding in the assay. This prevented assays at high substrate concentration being performed. Based on the kinetic data, we estimate that the $K_{\mathrm{m}}$ with Fru-1-P for both enzymes was well over $10 \mathrm{mM}$.

Surprisingly, none of the UGPases showed activity with Gal-1-P. This was in contrast to our earlier study (Decker et al., 2012), where the activity of barley UGPase with Gal-1-P at $0.5 \mathrm{mM}$ was $12 \%$ of that with Glc-1-P. As the pyrophosphorylase activities were tested at $1 \mathrm{mM}$ concentration of each of the sugar-1-phosphates (Figure 1), it was still possible that Gal-1-P would be reactive at higher concentrations. Indeed, as shown in Supplementary Figure S2, barley UGPase showed traces of activity when assayed with $10 \mathrm{mM}$ Gal-1-P, but the rate was at most $0.14 \%$ of that with $10 \mathrm{mM}$ Glc-1-P. To address this issue further, we have tested Gal-1-P batches from three sources: Sigma-Aldrich, Carbosynth and from Dr. Motomitsu Kitaoka (Ibaraki, Japan). In all cases, the activity with Gal-1-P was exceedingly low and almost linearly correlated with concentration of this substrate (up to $10 \mathrm{mM}$ tested) (Supplementary Figure S2). This suggested that the $K_{\mathrm{m}}$ with Gal-1-P was very high, likely over $10 \mathrm{mM}$. This should be compared to the UGPase activity with Glc-1-P, and the $K_{\mathrm{m}}$ with this substrate of $0.45 \mathrm{mM}$ (Supplementary Figure S2).

\section{Activities of USPase with Different Sugar Phosphates and NTPs}

Compared to UGPase, the activity of USPase showed much wider substrate preferences (Figure 2). The enzyme was most active with Gal-1-P, but its activity with Glc-1-P, galacturonic acid-1-P (GalA-1-P) and arabinose-1-P (Ara-1-P) was at least $60 \%$ of that shown with Gal-1-P. In all cases, UTP served as a more or less specific second substrate. Whereas the $K_{\mathrm{m}}$ values with Gal-1-P, Glc-1-P, Ara-1-P, GlcA-1-P and xylose-1-P (Xyl-1-P) (the last two compounds were not tested in the present study) have already been published for Arabidopsis USPase (Supplementary Table S2), in the present study we determined also its $K_{\mathrm{m}}$ with GalA-1-P; this $K_{\mathrm{m}}$ was $1.3 \mathrm{mM}$ (Supplementary Figure S3). Compared to already reported (also for Arabidopsis USPase) $K_{\mathrm{m}}$ values with GlcA-1-P of about 0.1 mM (Litterer et al., 2006b; Kotake et al., 2007) (Supplementary Table S2), this implies that USPase has considerably higher affinity for GlcA-1-P, when compared to that for GalA-1-P.

\section{Activities of UAGPase2 with Different Sugar Phosphates and NTPs}

Based on data in Figure 3, UAGPase2 reacted only with GlcNAc-1-P and GalNAc-1-P and, to some extent, with Glc-1-P. Similar to UGPase and USPase, in all cases UTP served as the only effective nucleotide donor. We have also observed very low rates with Fru-1-P, when GTP was the second substrate, but the significance of this is unclear. Earlier study on Arabidopsis UAGPase 2 also reported highest rates with GlcNAc-1-P and GalNAc-1-P, and some activity with Glc-1-P (Yang et al., 2010), but they did not report $K_{\mathrm{m}}$ values with GalNAc-1-P nor Glc-1-P. Thus, in the present study, the determined $K_{\mathrm{m}}$ value of UAGPase2 with GalNAc-1-P was $1.0 \mathrm{mM}$ (Supplementary Figure S4A), which was about 5-fold higher than that reported for Arabidopsis UAGPase2 with GlcNAc-1-P ( $K_{\mathrm{m}}$ of $\left.0.18 \mathrm{mM}\right)$ (Yang et al., 2010). This suggested that GlcNAc-1-P would be the preferred in vivo substrate. The same conclusion can be reached when comparing $K_{\mathrm{m}}$ values with UTP when GalNAc-1-P or GlcNAc-1-P served as the second substrate: $3 \mathrm{mM}$ (Supplementary Figure S4B) vs. 0.2 mM (Yang et al., 2010), respectively. 


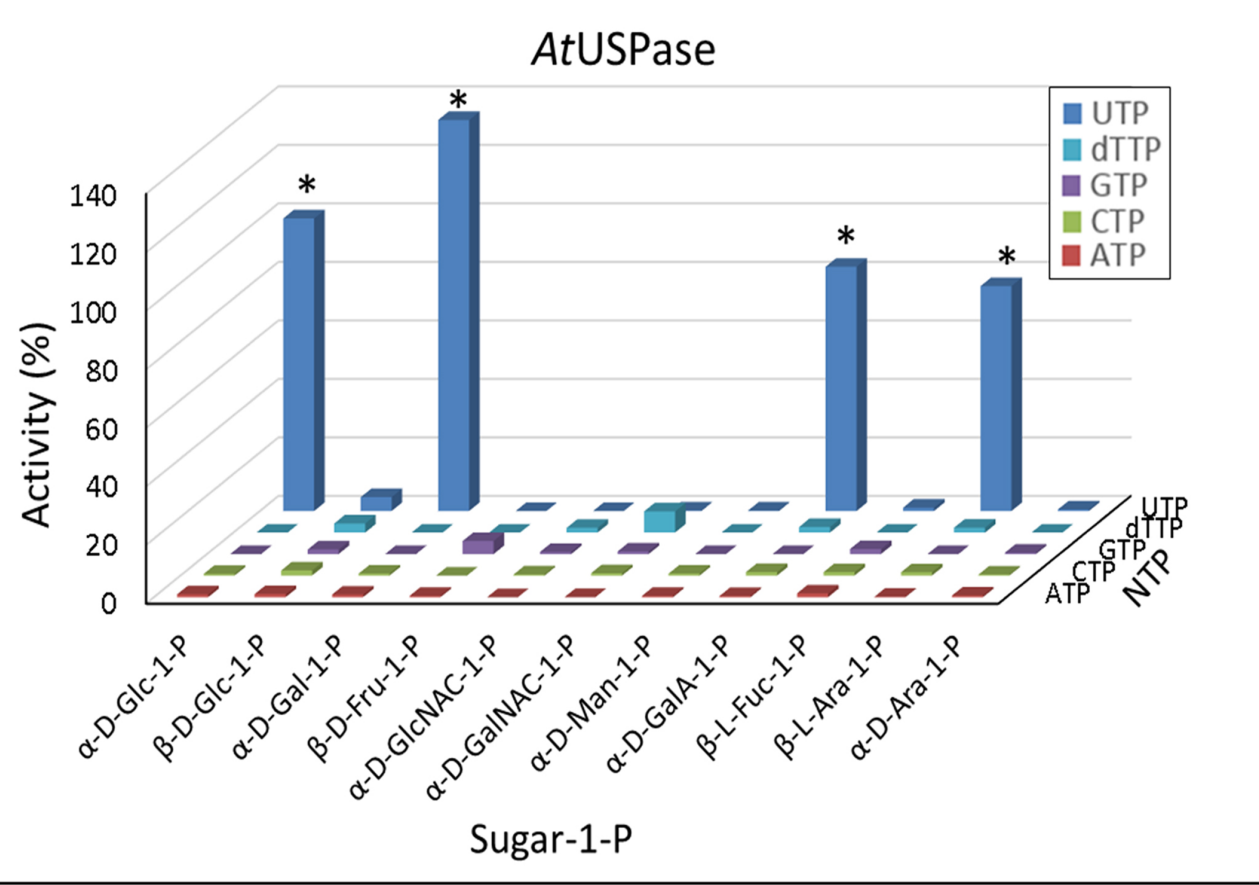

FIGURE 2 | Substrate specificity of Arabidopsis USPase. Each assay contained a sugar-1-P (at 1 mM) and a nucleoside triphosphate (at 1 mM). 100\% of activity refers to 55 units/mg protein. * indicates significant (Student's test) differences from background $(n=2, p<0.05)$.

The determined $K_{\mathrm{m}}$ values for UAGPase2 with Glc-1-P and UTP (forward reaction) were 3.2 and $0.4 \mathrm{mM}$, respectively, and with UDP-Glc and PPi (reverse reaction) - 0.21 and $0.32 \mathrm{mM}$, respectively (Supplementary Figure S5). Given its relatively high $K_{\mathrm{m}}$ with Glc-1-P, Arabidopsis UAGPase2 is rather unlikely to contribute to UDP-Glc formation in vivo, especially that plants contain both UGPase and USPase which have low $K_{\mathrm{m}}$ and high activity with Glc-1-P. Since Glc-1-P is the common substrate (along with UTP) for UAGPase2, UGPase and USPase, assays of Glc-1-P/UTP-dependent activity in crude plant extracts would reflect the sum of activities of the three pyrophosphorylases. The same concerns assays of UDP-Glc/PPi-dependent activity in crude extracts.

\section{Predicting and Verifying other Substrates for UGPase, USPase and UAGPase2}

By screening pyrophosphorylase activities against 11 sugar-1phosphates, we found that UGPase reacted only with Glc-1-P and Fru-1-P (Figure 1), whereas USPase and UAGPase2 used respectively four and three of those compounds as substrates (Figures 2, 3). In an effort to identify other substrates, we used QSAR approach, based on Free-Wilson model (Free and Wilson, 1964). The model takes into account properties of compounds related to those that were found active in a given system (see Material and Methods) and predicts whether they would be active as well solely based on similarity to active compounds. In our analysis, different configurations and types of substitutions to a nucleotide-sugar molecule (mainly sugar) were scored, allowing a simplified prediction of the activity of a given derived nucleotidesugar with a given pyrophosphorylase.

For UGPase, which was only active with Glc-1-P and, to some extent, Fru-1-P (Figure 1), no new potential substrates could be predicted. However, since the reactivity of UGPase with Fru-1-P was surprising, other fructose-phosphates were selected, based on commercial availability, and tested as potential substrates. Thus, in a separate study, the activity of barley UGPase was assayed with $1 \mathrm{mM}$ concentrations of Fru-1-P, Fru-2-P, Fru-6-P, and Fru-2,6-bisP. The results demonstrated that, in addition to Fru-1-P, the UGPase had considerable activity $(27 \%$ compared to the rate with Glc-1-P) with Fru-2-P (Supplementary Figure S6). Similar to Fru-1-P, the activity with Fru-2-P was characterized by a very high $K_{\mathrm{m}}$ (over $10 \mathrm{mM}$ ) (Supplementary Figure S6B).

For USPase, the QSAR analysis predicted that $\alpha-\mathrm{D}$-fucose-1-P ( $\alpha$-D-Fuc-1-P) and $\alpha$-D-quinovose-1-P (Qui-1-P) could serve as substrates for this enzyme (Figure 4A). Since Qui-1-P is not commercially available, in subsequent studies we could test only the other compound. Assays of USPase with $\alpha$-D-Fuc-1-P revealed that this compound indeed served as substrate, and the activity was about $20 \%$ of that with Glc-1-P (Figure $4 \mathbf{B}$ ). The reactivity of USPase with $\alpha$-D-Fuc-1-P was earlier inferred by Liu et al. (2013) who used Arabidopsis USPase as one of three "coupling enzymes" to produce UDP- $\alpha$-D-Fuc from $\alpha$-D-Fuc. In the present study, by directly measuring USPase activity with $\alpha$-D-Fuc-1-P, we were able to determine $K_{\mathrm{m}}$ for this compound (Supplementary Figure S3). The $K_{\mathrm{m}}$ was relatively high at $3.4 \mathrm{mM}$. 


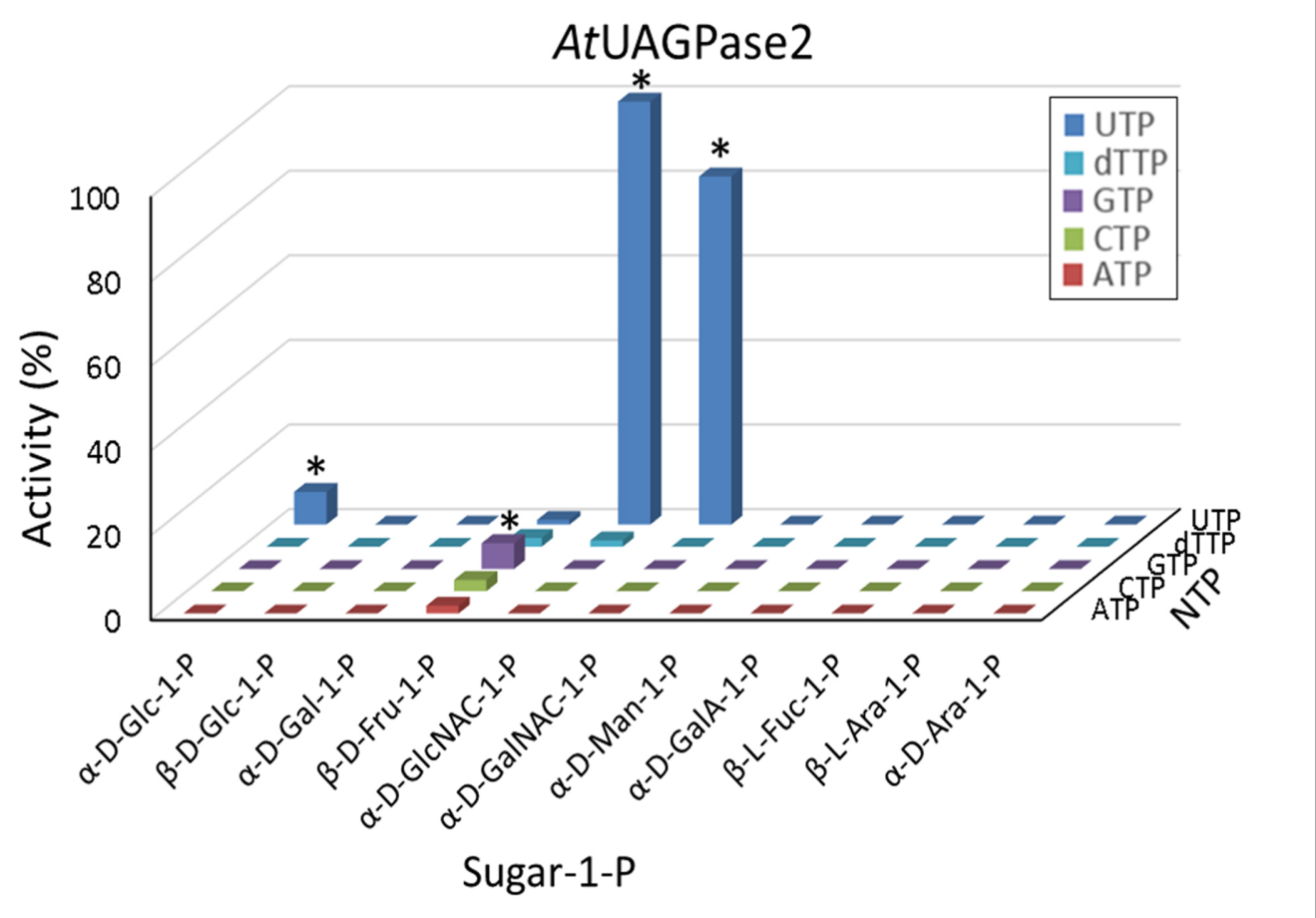

FIGURE 3 | Substrate specificity of Arabidopsis UAGPase2. Each assay contained a sugar-1-P (at 1 mM) and a nucleoside triphosphate (at 1 mM). 100\% of activity refers to 6.5 units/mg protein. ${ }^{*}$ indicates significant (Student's test) differences from background $(n=2, p<0.05)$.

The QSAR analyses of possible alternative substrate(s) for UAGPase2 revealed only GlcN-1-P as a plausible candidate (Figure 4C). However, assays of UAGPase2 with this compound yielded no activity (Figure 4D). This probably underscores the importance of the acetyl group and/or the secondary amine in GlcNAc-1-P, one of true substrates of plant UAGPase. The lack of activity with GlcN-1-P was also reported for UAGPase from Aspergillus fumigatus (a fungus) (Fang et al., 2013).

\section{Structure-Function Relationships for Sugar-1-Phosphates as Substrates of UGPase, USPase and UAGPase2}

The chemical structures of sugar-1-phosphates considered in this analysis are shown in Figure 5. The sugars were divided into those in the pyranose and furanose (only Fru-1-P) form, and listed according to orientation of the hydroxyl group at carbon 4 (C4) (equatorial or axial) and according to whether they were active or inactive with any of the UDP-sugar producing pyrophosphorylases. Please note that Fru-1-P is presented here as $\beta$-D furanose (Figure $5 \mathrm{C}$ ), but the exact structure of this compound in its active form in solution, i.e., whether it is in $\alpha$ or $\beta$ configuration and/or whether in furanose or pyranose form, is unknown. The list includes also Qui-1-P, which was suggested by the QSAR model to serve as one of USPase substrates (Figure 4A), but was not tested. Omitted from the diagram were certain C3 epimers of Glc-1-P (allose-1-P, altrose-1-P, gulose-1-P,
idose-1-P and talose-1-P) which are not commercially available and for which there are no described roles in plants.

As shown in Figure 5A, UGPase accepts phosphorylated hexoses which have a Glc-type configuration of hydroxyl groups at C1-4 and C6, but allowing also for a trace activity with Gal-1-P, a C4 epimer of Glc-1-P. Concerning USPase, which can react with several phosphorylated hexoses and pentoses as substrates, its specificity is likely determined by the equatorial hydroxyl group at $\mathrm{C} 2$ of the sugar molecule (Figure 5A). This requirement could be further verified if the reactivity with Qui-1-P were tested. In comparison to UGPase and USPase, UAGPase2 reacts only with phosphorylated hexoses with an $\mathrm{N}$-acetyl group at C2 of Glc or Gal moieties, but can also react with Glc-1-P (but not Gal-1-P), where the $N$-acetyl moiety at $\mathrm{C} 2$ is replaced by a hydroxyl group (Figure 5A). The sugars that were inactive for all three pyrophosphorylases are epimers of the active sugars, and they differ from them in the stereochemical attachment of hydroxyl groups at either C1, C2, or C3 position (Figure 5B).

\section{Conservation of UDP-Sugar Binding Sites in Plant UGPase, USPase and UAGPase2}

Besides their substrate specificity, we have also analyzed details of UDP-sugar binding to crystal structure of Arabidopsis UGPase1 (McCoy et al., 2007) and to homology-modeled structures of Arabidopsis USPase and Arabidopsis UAGPase2 bound to a high affinity substrate (UDP-Glc for UGPase and USPase; and 


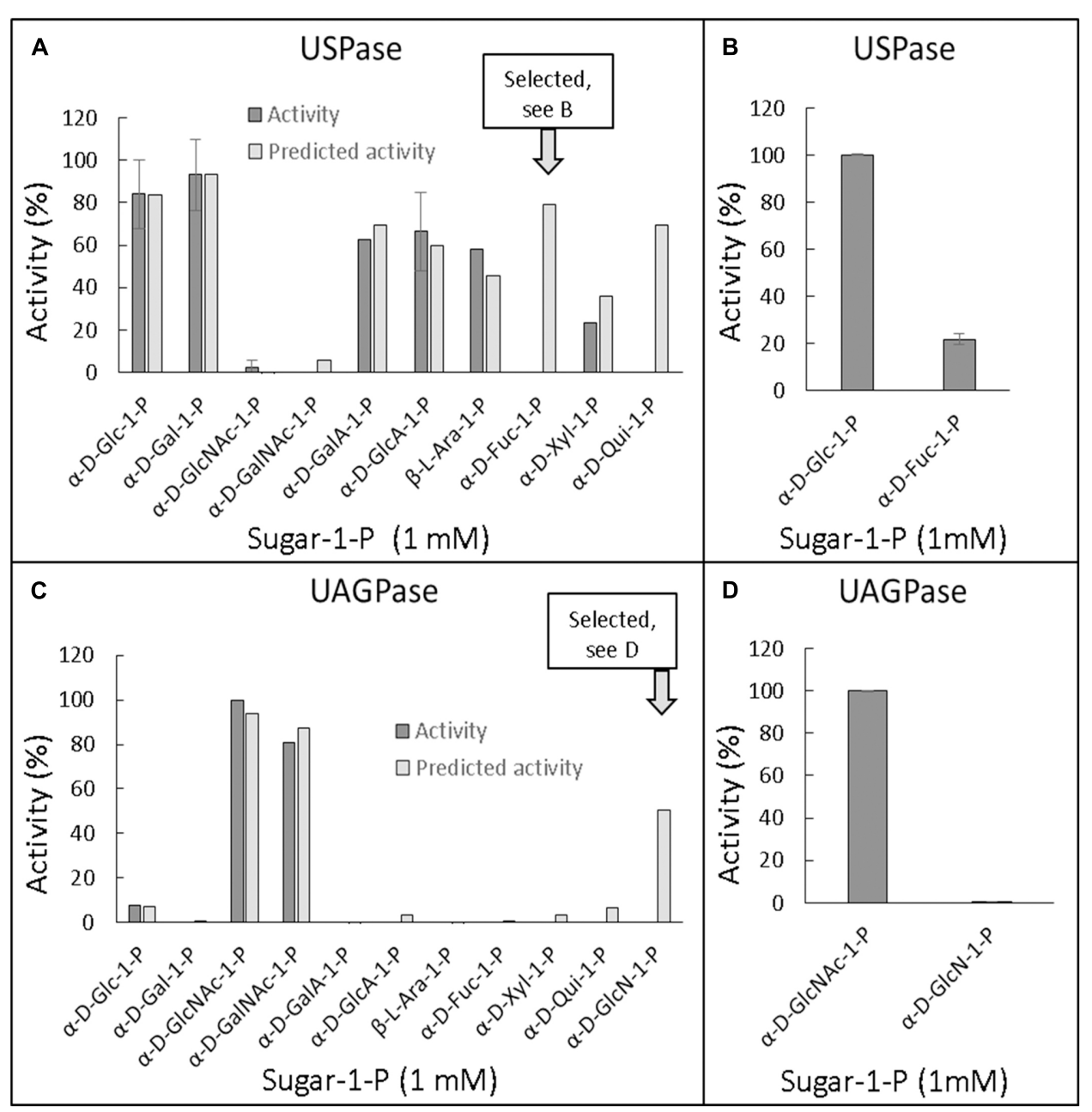

FIGURE 4 | Quantitative structure activity relationship (QSAR) of Arabidopsis USPase (A,B) and Arabidopsis UAGPase2 (C,D) with different sugar-1-phosphates as substrates. (A,C): Determined (also literature-based, referenced in Supplementary Table S2) data for USPase and UAGPase activity, respectively, were used to construct a model (Free and Wilson, 1964) for possible other substrates, using 1D-descriptors (see Materials and Methods). (B,D): Verification of the predicted substrate activity. All assays, done in two replicates, contained $1 \mathrm{mM}$ sugar-1-P and $1 \mathrm{mM}$ UTP.

UDP-GlcNAc for UAGPase) (Supplementary Figure S7). The homology models were respectively based on crystal structures of Leishmania USPase (Dickmanns et al., 2011) and human UAGPase2 (Peneff et al., 2001). As seen in Supplementary Figure S7, the relative positioning of UDP-sugar and at least some of the interacting amino acid (aa) residues (via hydrogen bonds and hydrophobically) appear similar for all three enzymes, but a more detailed comparison was needed. To do so, we have overlayed substrate binding pockets of AtUGPase1 and AtUSPase (Figure 6A) and those of AtUGPase1 and AtUAGPase2 (Figure 6B). To simplify representations of the active sites, only AtUGPase1 with bound UDP-Glc is shown, but aa residues in an equivalent position in the compared structure (USPase or UAGPase) are encircled in red. Also, the numbers for aa reflect those of UGPase aa sequence.

In both UGPase/USPase and UGPase/UAGPase cases, there appears to be high degree of conservation of aa residues interacting with the nucleotide part of UDP-sugar. All three pyrophosphorylases contain amino acid residues corresponding to Gly87, Met134, Gln162 Pro189 and Gly191 of UGPase in equivalent positions, which may interact/ stabilize the uridine portion of the UDP-sugar substrate. In some other cases shown in Figure 6, the three pyrophosphorylases share similar aa (but not identical) in equivalent positions which may be involved in stabilizing/interacting with given parts of the UDP-sugar substrate. For instance, ribose part of uridine is stabilized/ interacts with either a leucine residue (Leu85 and Leu130 for UGPase and UAGPase2, respectively) or with valine (Val134 for USPase) (Supplementary Figure S7).

Residues in proximity of the sugar moiety are less conserved when comparing UGPase, USPase and UAGPase2. They include, for instance, an Asn-X-Asn motif in the proximity of the C2 and/or C3 of the sugar substrate of UGPase and USPase, but not UAGPase2 (Figure 6). Also, His192 and His 254 in 


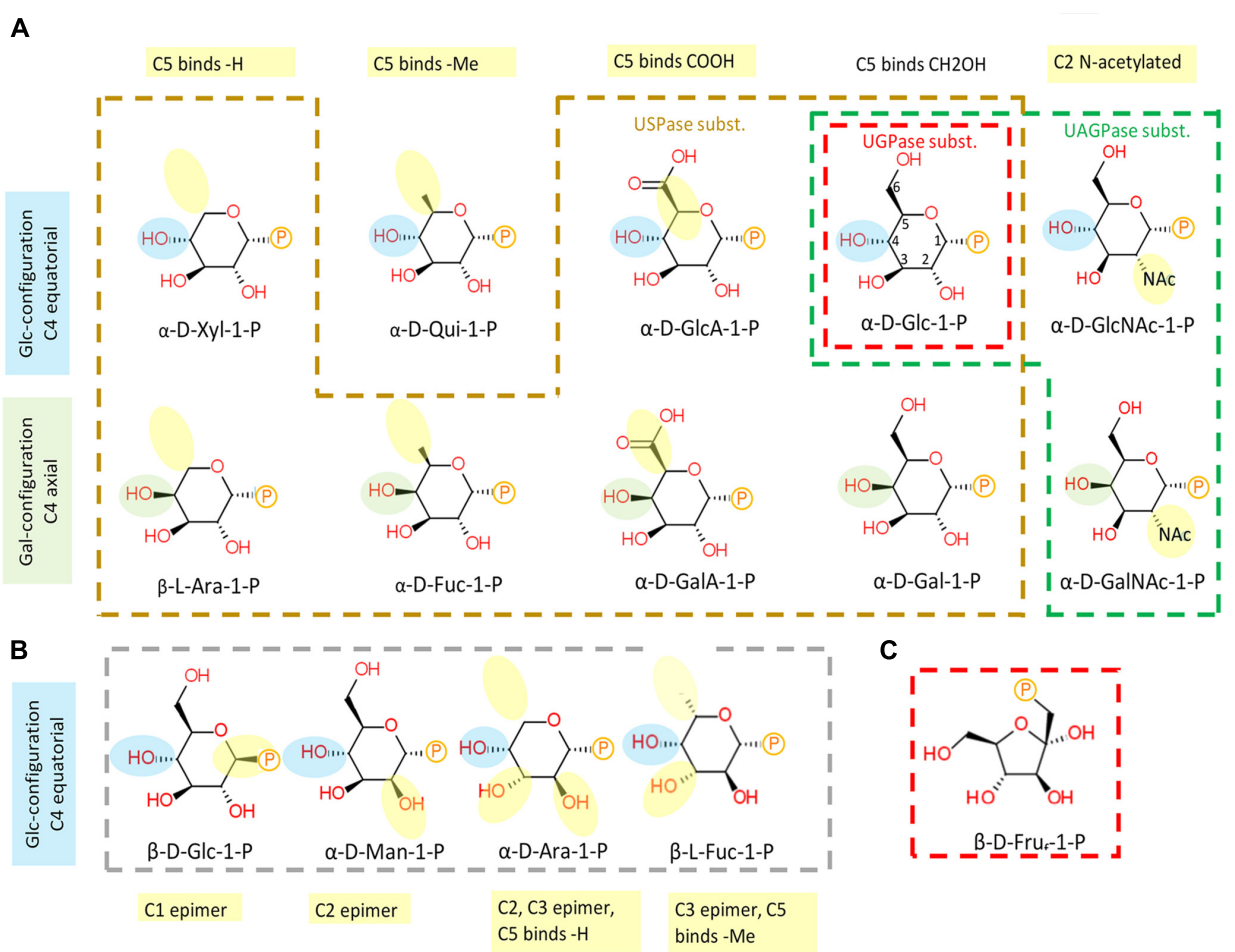

FIGURE 5 | Substrate structure-activity relationship of plant UDP-sugar producing pyrophosphorylases. (A) Sugar-1-phosphates which are substrates for respective enzymes are encircled in different colors: UGPase - red, USPase - orange, and UAGPase2 - green. (B) Inactive compounds are encircled in gray. (C) Fru-1-P drawn in furanose form. Numbering of $\mathrm{C}$-atoms are shown on $\alpha$-D-Glc-1-P. Phosphate group is shown as P. Blue and green highlights of -OH at C4 refer to equatorial (Glc-like) and axial (Gal-like) configurations, respectively. Yellow highlight refers to modifications in relation to Glc.

UGPase and USPase, respectively, occupy the space in which Asn250 is present in UAGPase2, i.e., in the proximity of the diphosphate and -NAc portions of the nucleotide sugar substrate (Supplementary Figure S7). For UGPase, the hydroxyl group at C4 of the substrate lies in proximity of a bulky aromatic nonpolar residue (Phe292), while in USPase or UAGPase2 it is located near a smaller aliphatic non-polar residue (Ile319 or Val316, respectively). Both Gly320 and Gly317 in USPase and UAGPase2, respectively, may form a hydrogen bond to the $C 4$ hydroxyl of the sugar moiety and have no equivalent in UGPase (Supplementary Figure S7).

It should be emphasized that details of UDP-sugar binding to plant USPase and UAGPase2 in Figure $\mathbf{6}$ are only approximate since their structures were modeled respectively on Leishmania and human proteins, which are evolutionarily distant from the corresponding plant proteins. For instance, Leishmania and Arabidopsis USPases share only 37\% identity, based on their aa sequences (Kleczkowski et al., 2011a), whereas human and Arabidopsis UAGPase proteins have about 43\% identity (Yang et al., 2010). The plant UGPase model, however, is based on crystal structure of Arabidopsis UGPase1, and thus represents a more accurate representation of the UDP-Glc binding to the enzyme. It should also be noted that these comparisons are based on rigid structures and do not take into account any local conformational changes that may occur during substrate binding and/or catalysis.

\section{Inhibitor Effects on Purified Enzymes}

In recent study (Decker et al., 2017), we have separately used purified UGPase and purified USPase to screen a chemical library for compounds affecting a given activity. Surprisingly, the identified componds inhibited both UGPase and USPase activities, probably reflecting similar aspects of active sites of those proteins (Kleczkowski et al., 2011b). Hit expansion analyses for one of the compounds, a salicylate derivative, yielded an analog, named $\mathrm{cmp} \# 6 \mathrm{D}$, which acted as efficient inhibitor of both enzymes, and was also active in vivo by inhibiting pollen germination (Decker et al., 2017). Since homology-modeled AtUAGPase2 tertiary structure is in many aspects similar to crystal structures of UGPase and USPase (McCoy et al., 2007; Yang et al., 2010; Dickmanns et al., 2011; Kleczkowski et al., 2011b), we tested whether cmp \#6D had any effect on activity of purified Arabidopsis UAGPase2. Indeed, cmp \#6D at $50 \mu \mathrm{M}$ inhibited UAGPase2 activity by $50 \%$ (Table 1), and the degree of inhibition was roughly comparable with that for UGPase and USPase.

In addition to effects of $\mathrm{cmp} \# 6 \mathrm{D}$, we have also tested UAGPase 2 sensitivity to an indolinone-derivative which was earlier identified as inhibiting UAGPase from Trypanosoma brucei (with Ki of $60 \mu \mathrm{M}$ ), but not human UAGPase (Urbaniak et al., 2013). This inhibitor (which we called cmp \#41) had no effect on Arabidopsis UAGPase 2 activity, even at a concentration as high as $0.4 \mathrm{mM}$ (data not shown), nor on activities of barley 


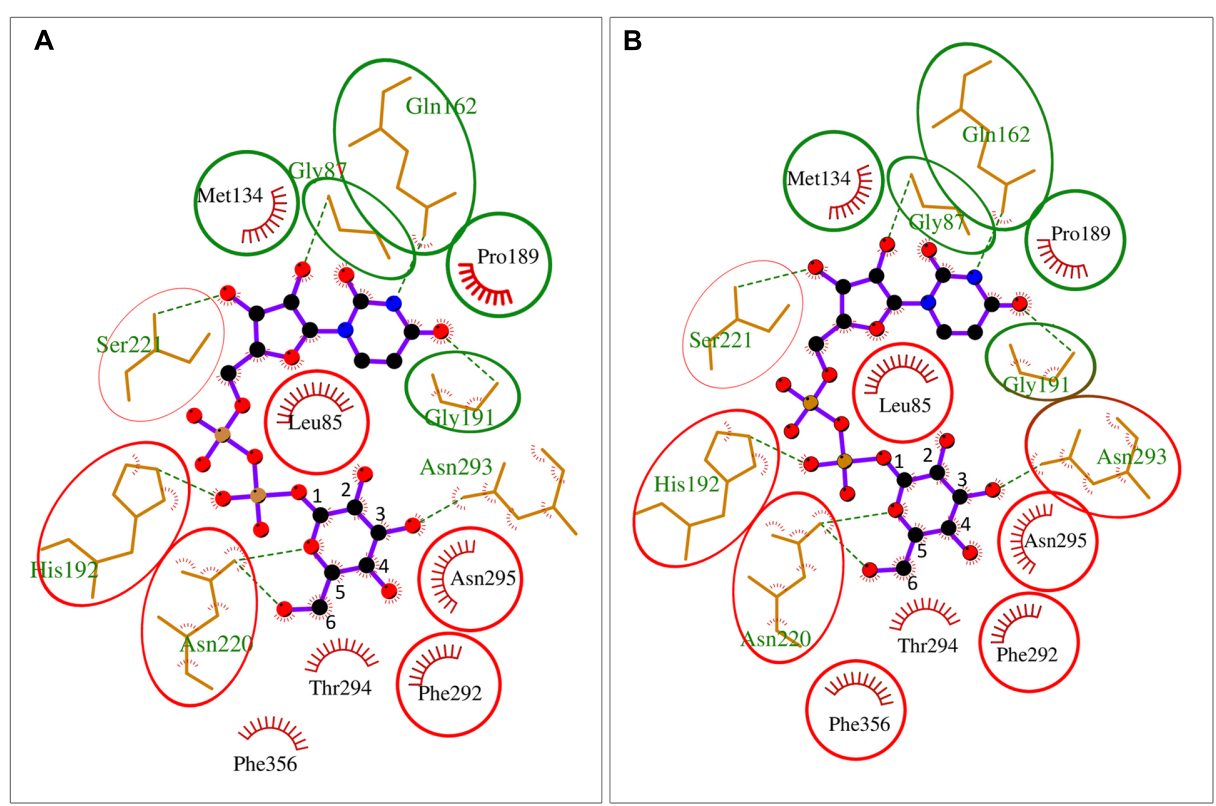

FIGURE 6 | Schematic representation of UDP-Glc binding to AtUGPase1 vs. AtUSPase (A) and AtUGPase1 vs. AtUAGPase2 (B). Names of aa which interact or are in proximity of UDP-Glc are in black (hydrophobic interaction) or green font (hydrogen bond). Numbers for aa refer to UGPase aa sequence. Green circles mark the same type of aa residues in the comparable position for all three enzymes. Red circles mark aa which have an equivalent aa counterpart for a given UGPase/USPase and UGPase/UAGPase comparison. The uncircled aa are only found in AtUGPase. Active sites of the pyrophosphorylases were analyzed and presented using LigPlot+, based on the resolved crystal structure of AtUGPase1 (2ICY) and homology models of AtUSPase and AtUAGPase2, based on crystal structures of Leishmania USPase (PDB 3OH4) (Dickmanns et al., 2011) and human UAGPase (PDB 1JV1), (Peneff et al., 2001), respectively.

UGPase and Arabidopsis USPase (Table 1). Until the crystal structure of Arabidopsis UAGPase2 is resolved, it is unknown whether the lack of inhibition by $\mathrm{cmp} \# 41$ is the result of any particular structural difference between the Trypanosoma and Arabidopsis UAGPase 2 proteins.

\section{DISCUSSION}

In photosynthetic tissues, pyrophosphorylases represent the most important mechanism of the production of a UDP-sugar, as they use UTP and sugar-1-P that are derived more or less directly from photosynthetic light reactions and the Calvin cycle, respectively. In sink tissues, the pyrophosphorylases

TABLE 1 | Inhibition of purified barley UGPase, Arabidopsis USPase and Arabidopsis UAGPase2 by $\mathrm{cmp} \# 6 \mathrm{D}$ and \#41.

\begin{tabular}{lccc}
\hline & \multicolumn{3}{c}{ Activity (\%) } \\
\cline { 2 - 4 } Enzyme & No inhibitor & $\mathbf{c m p ~ \# 6 D}$ & $\mathbf{c m p}$ \#41 \\
\hline UGPase & $100 \pm 2$ & $37 \pm 2$ & $91 \pm 8$ \\
USPase & $100 \pm 2$ & $56 \pm 6$ & $96 \pm 6$ \\
UAGPase2 & $100 \pm 2$ & $50 \pm 8$ & $100 \pm 12$
\end{tabular}

Assays were carried out in the pyrophosphorylytic direction (see Material and Methods). The 100\% activities for UGPase, USPase and UAGPase corresponded to 405,12 and 7 units $/ \mathrm{mg}$, respectively. Inhibitors were at $50 \mu \mathrm{M}$. Data are mean $\pm S E(n \geq 2)$. predominantly use sugar-1-P derived from hydrolysis of sucrose by either SuSy or invertase. In those tissues, SuSy may have a more prominent role, since it uses transported/accumulated Suc to produce UDP-Glc as one of its substrates (Huber and Akazawa, 1986; Kleczkowski, 1994b), which then serves as direct precursor to e.g., cellulose formation (Fujii et al., 2010). In cereal seed endosperm, the SuSy-derived UDP-Glc may also provide a metabolic link between sucrose hydrolysis and starch synthesis, being used in the reverse reaction of UGPase to produce Glc-1-P, which then serves as substrate for cytosolic AGPase (Kleczkowski, 1996). Whereas many UDP-sugars can be derived from UDP-Glc via nucleotide sugar interconversion reactions (e.g., UDP-Glc epimerase or UDP-Glc dehydrogenase) (Kleczkowski and Decker, 2015), the exact contributions of these different reactions to the nucleotide-sugar pools are still unclear, although it appears that de novo pathways dominate (Sharples and Fry, 2007). The role of USPase has already been demonstrated in Arabidopsis where it participates in the metabolism of UDP-Ara and UDP-Xyl, based on analyses of transgenic plants with knocked-down USPase activity (Geserick and Tenhaken, 2013a,b). In addition, these and other studies on transgenic plants with impaired/knocked out USPase, UGPase and UAGPase activities (e.g., Schnurr et al., 2006; Kotake et al., 2007; Meng et al., 2009b; Park et al., 2010; Chen et al., 2014) have underlined the importance of these enzymes during reproductive phases of plant development, in most cases by affecting cell wall polysaccharide formation (reviewed in Kleczkowski and Decker, 2015). 


\section{Revisiting Substrate Specificity of UDP-Sugar Producing Pyrophosphorylases}

Barley and Arabidopsis UGPases as well as Arabidopsis USPase and Arabidopsis UAGPases have been the most extensively studied representatives of the plant UDP-sugar producing family (Ritter et al., 1996; Kleczkowski et al., 2004, 2011a; Litterer et al., 2006b; Meng et al., 2008; Yang et al., 2010) and thus we used them as representative enzymes to reexamine substrate specificity of this group of enzymes. Moreover, this is the first study where activities and kinetics of purified UGPases, USPases and UAGPase are examined side by side, using similar assay systems.

Both barley UGPase and the two Arabidopsis UGPases had very similar substrate specificity, with Glc-1-P and UTP acting as the most active substrates, but they had also a $7-20 \%$ activity with Fru-1-P, regardless of the NTP used (Figure 1). This reactivity with different NTPs, not only UTP, was unique for Fru-1-P and the UGPases, and was not observed for any other sugar-1-P serving as substrate for either UGPase, USPase or UAGPase. The Fru-1-P- and UTP-dependent activity was earlier reported for an isozyme/isoform of potato tuber UGPase (from a coldsweetening resistant cultivar) (Gupta and Sowokinos, 2003).

The Fru-1-P-dependent activity was observed for the UGPases, but not for USPase nor UAGPase activities (Figures 2, 3). Interestingly, a USPase from a bacterium Thermus caldophilus was also shown not to have any activity with Fru-1-P, but it was activated by this compound (Kim et al., 1999). The $K_{\mathrm{m}}$ of barley UGPase with Fru-1-P was high (over $10 \mathrm{mM}$ ) (Supplementary Figure S1), so it is likely that Glc-1-P ( $K_{\mathrm{m}}$ of $0.33 \mathrm{mM}$; Decker et al., 2012) would largely outcompete Fru-1-P as an in vivo substrate. The origin of Fru-1-P in plants is also problematic, even though this compound was found in several plant species (e.g., Graham and ap Rees, 1965; Geigenberger et al., 2004), similar to UDP-Fru (Feingold, 1982). Fru-1-P can be produced either from Fru by a ketohexokinase, a distinct type of hexokinase active in animal liver (Geigenberger et al., 2004), or by an aldolase (B-type), also found in liver, which uses glyceraldehyde and dihydroxyacetone phosphate as substrates, or by a phosphofructomutase-like activity, producing Fru-1-P from Fru-6-P, as reported for Aeromonas hydrophila, a species belonging to Proteobacteria (Binet et al., 1998). To our knowledge, neither of these enzymes are present in plants, suggesting yet unknown mechanism of Fru-1-P formation there.

UGPase was also active with Fru-2-P, again with a high $K_{\mathrm{m}}$ (over $10 \mathrm{mM}$ ) (Supplementary Figure S6). Similar to UDP-(1)-Fru, the rationale for producing and usage of UDP-(2)-Fru in vivo is obscure. On the other hand, chemical analyses of UDP-Fru extracted from Jerusalem artichoke suggested that most of it is in the UDP-(2)-Fru form rather than that of UDP-(1)-Fru (Taniguchi and Nakamura, 1972). Fru-(2)-glycosidic bonds are found in some naturally occurring molecules, e.g., sucrose (Daudé et al., 2012). UDP-2-Fru would resemble the UDP-sugars used by glycosyltransferase reactions (Lairson et al., 2008), as the reported nucleotide sugars, both furanoses and pyranoses, are commonly activated on anomeric carbon (e.g., by pyrophosphorylases) (Bar-Peled and O'Neill, 2011). Fru-2-P, the substrate for formation of UDP-(2)-Fru by UGPase, could originate from C-6 dephosphorylation of Fru-2,6-bisP; such an activity has been reported in both plant and yeast extracts (Larondelle et al., 1989).

The reactivity of UGPase with fructose-phosphates requires further studies. Structures of products of this reaction need to be determined, using, e.g., mass and NMR spectrometry, to address the question which of the sugar carbons is activated during the reaction. An artefactual phosphatase activity of UGPase, but not USPase nor UAGPase2, in the presence of certain fructosephosphates is also a possibility.

Unlike in our previous report (Decker et al., 2012), the activity of barley UGPase was negligible with Gal-1-P (Supplementary Figure S2). This difference could be ascribed to the fact that in the earlier study we had used different batch of Gal-1-P, and we cannot rule out that it was contaminated by Glc-1-P. Based on our present results, using batches of Gal-1-P from three different sources, the results strongly indicate that the Gal-1-P-dependent activity of UGPase is unlikely to have any significance in vivo, due to negligible rates and very high $K_{\mathrm{m}}$ for this compound. Extremely low activities with Gal-1-P were also reported for Arabidopsis UGPase1 and UGPase 2 isozymes (Meng et al., 2008). In contrast to UGPase, USPase can effectively carry out the same reaction at a much lower concentration of Gal-1-P (Figure 2), having $K_{\mathrm{m}}$ of $0.27 \mathrm{mM}$ (Kotake et al., 2007) (Supplementary Table S2). Nevertheless, activities with Gal-1-P have sporadically been reported for plant UGPases (Gupta and Sowokinos, 2003; Kim and Ahn, 2013), including the chloroplastic UGPase isozyme (Okazaki et al., 2009).

Previous studies of non-recombinant barley UGPase (purified from barley malt) (Ritter et al., 1996) demonstrated that the enzyme had a somewhat less strict substrate specificity (i.e., low activity with GlcA-1-P and GalA-1-P, in addition to that with Glc-1-P), when compared to the recombinant (expressed from procaryote hosts) enzymes used in the present study. These differences in substrate specificity could perhaps be explained by post-translational modifications which are specific for eucaryotes/plants (Sauerzapfe et al., 2008) and/or simply by differences in assay conditions, e.g., 1 vs. $2 \mathrm{mM}$ NTP and 5 vs. $10 \mathrm{mM} \mathrm{MgCl}_{2}$ for this study and Ritter's et al., (1996), respectively. However, it should be emphasized that, in terms of substrate specificity, plant UGPases are very different from mammalian UGPases which, in addition to Glc-1-P, have relatively high activities with several other sugar-1-phosphates, producing corresponding UDP-sugars (Knop and Hansen, 1970; Ritter et al., 1996). In this respect mammalian UGPases resemble USPases. Mammals apparently lack USPase (Kleczkowski et al., 2011a), and thus their UGPases may have evolved to compensate for that by extending their range of sugar-1-phosphates used as substrates.

As in earlier studies on substrate specificity of plant USPases (Kotake et al., 2004, 2007; Dai et al., 2006; Litterer et al., 2006a,b), Arabidopsis USPase was found as highly promiscuous with respect to various sugar-1-phosphates as substrates, with the highest activity shown with Gal-1-P, Glc-1-P, GalA-1-P, 
and L-Ara-1-P (Figure 2). Somewhat similar properties were reported for USPases from protozoan pathogens Leishmania and Trypanosoma (Damerow et al., 2010; Yang and Bar-Peled, 2010). However, the activity with GalA-1-P for Arabidopsis USPase was only seldom reported (Yang et al., 2009), and its $K_{\mathrm{m}}$ value was unknown. We have now determined $K_{\mathrm{m}}$ of Arabidopsis USPase with GalA-1-P, as shown in Supplementary Figure S3A. This relatively low $K_{\mathrm{m}}$ of $1.3 \mathrm{mM}$ is comparable to $K_{\mathrm{m}}$ of $2.3 \mathrm{mM}$ determined with GalA-1-P for pea USPase (Ohashi et al., 2006), suggesting that plant USPase (in addition to UDP-GlcA epimerase) could be involved in the production of UDP-GalA in vivo, e.g., during recycling of GalA released during cell wall restructuring.

Arabidopsis USPase was reactive with $\alpha$-D-Fuc-1-P (Supplementary Figure S3B), but not with $\beta$-L-Fuc-1-P (Figure 2). The reactivity with $\alpha$-D-Fuc-1-P was inferred from QSAR analyses (Figure 4A) and then confirmed experimentally (Figure 4C). Fuc moiety can be found in cell wall polysaccharides and in sugar components of glycoproteins, and it is usually present there in the $\alpha$-L-Fuc form (Scheible and Pauly, 2004; Harholt et al., 2010; Scheller and Ulvskov, 2010). The activated form of Fuc used by glycosyltransferases is believed to be GDP- $\beta$ L-Fuc, rather than UDP- $\alpha$-D-Fuc, and in plants it is synthesized from GDP- $\alpha$-D-mannose (GDP- $\alpha-D-M a n)$ via combined activities of specific dehydratase and epimerase/reductase or by a bifunctional kinase/GDP-Fuc pyrophosphorylase protein that converts $\beta$-L-Fuc to $\beta$-L-Fuc-1-P, and then to GDP- $\beta$-L-Fuc (Kotake et al., 2008). The formed GDP- $\beta$-L-Fuc may then subsequently be used by an inverting-type of a glycosyltrasferase, which inverts anomeric configuration of the sugar residue upon transferring it to a donor molecule (Lairson et al., 2008).

In contrast to GDP- $\beta$-L-Fuc, very little is known about the origins and the roles of UDP- $\alpha$-D-Fuc in plants. As strong USPase knock-down had no changes in cell wall nor leaf soluble fucose content (Geserick and Tenhaken, 2013a), UDP- $\alpha$-D-Fuc may not be a key player in fucose cycling or cell wall formation, but perhaps may be involved in secondary metabolism. Addition of a synthetically prepared UDP- $\alpha$-D-Fuc to leaf extracts was reported to lead to incorporation of the Fuc molecule to cardenolide aglycones, a group of secondary metabolites (Faust et al., 1994), but the resulting compounds were $\beta$-D-fucosylated rather than $\alpha$-D-fucosylated, suggesting the involvement of an inverting-type of a glycosyltrasferase. Plants contain many such glycosyltransferases (Lairson et al., 2008), but to our knowledge none has yet been identified which carries the inversion when using UDP- $\alpha-D-F u c$ as a substrate. Considering the structural similarities between $\alpha$-D-Fuc and $\alpha$-D-Gal (Figure 5), such an enzyme can perhaps be found among the UDP- $\alpha$-D-Gal-utilizing glycosyltransferases. Given its relatively low $K_{\mathrm{m}}$ of $3.4 \mathrm{mM}$ with $\alpha$-D-Fuc-1-P (Supplementary Figure S3B), USPase may perhaps represent yet another mechanism in the pathway from Fuc to a fucosylated product molecule.

Arabidopsis UAGPase2 was found to react preferentially with GlcNAc-1-P and GalNAc-1-P, with UTP acting as the sole NTP substrate (Figure 3), confirming earlier data on this enzyme (Yang et al., 2010). In this respect, Arabidopsis
UAGPase2 is similar to human UAGPase (Peneff et al., 2001), but not to UAGPases from Aspergillus fumigatus (a fungus) and Trypanosoma brucei (single-celled eukaryotic pathogen), which do not react with GalNAc-1-P as substrate (Stokes et al., 2008; Fang et al., 2013). Because of its relatively low $K_{\mathrm{m}}$ values with GlcNAc-1-P (0.18 mM, Yang et al., 2010) and GalNAc-1-P (1.0 mM, Supplementary Table S2), it appears that UAGPase2 is the key activity "activating" both of these $N$-acetyl hexoseamines, so they can be used for glycosylation events in plants (Bar-Peled and O'Neill, 2011). However, the enzyme had a relatively high $K_{\mathrm{m}}$ for UTP ( $3 \mathrm{mM}$ ) when GalNAc-1-P served as the second substrate (Supplementary Figure S4), which should be compared with $K_{\mathrm{m}}$ of $0.2 \mathrm{mM}$ for UTP when GlcNAc-1-P was the other substrate (Yang et al., 2010). This implies that the GlcNAc-1-P-dependent activity would be favored over that with GalNAc-1-P. This is consistent with earlier data for the reverse reaction of Arabidopsis UAGPase2, where $K_{\mathrm{m}}$ value with UDP-GlcNAc was over 10 times lower than that with UDP-GalNAc (0.07 mM vs. $0.81 \mathrm{mM}$, respectively) (Yang et al., 2010). To assure sufficient production of the latter, plants may have an additional mechanism in the form of an epimerase which directly converts UDP-GlcNAc to UDP-GalNAc (Zhang et al., 2006; Furo et al., 2015).

The UAGPase2 enzyme was also able to utilize Glc-1-P as a substrate (Figure 3). It is, however, rather unlikely to be involved in a large scale UDP-Glc synthesis in vivo, given its relatively low rates (Yang et al., 2010) and high $K_{\mathrm{m}}$ of $3.2 \mathrm{mM}$ with this compound (Supplementary Figure S5) and, even more so, because both UGPase and USPase have much higher affinities for Glc-1-P as substrate (Supplementary Table S2). On the other hand, the relatively low $K_{\mathrm{m}}$ values for substrates of the reverse reaction (Supplementary Figure S5) make it feasible for UAGPase2 to be involved in Glc-1-P formation from UDP-Glc, similar to UGPase and USPase reaction.

$K_{\mathrm{m}}$ values determined with Glc-1-P for the three pyrophosphorylases (Supplementary Table S2) should be compared with Glc-1-P concentrations found in the cytosol of plant tissues, i.e., $0.07 \mathrm{mM}$ for barley endosperm (Tiessen et al., 2012) and $0.05 \mathrm{mM}$ in the cytosol of developing potato tubers (Farré et al., 2001). To our knowledge, there are no data on cytosolic concentrations of other sugar-phosphates, but they may be on the same order of magnitude as Glc-1-P, since their metabolism is frequently directly or indirectly linked to that of Glc-1-P (Kleczkowski and Decker, 2015). Thus, given $K_{\mathrm{m}}$ values for sugar-1-phosphates on the order of 0.1-10 mM (Supplementary Table S2), the in vivo activities of the pyrophosphorylases are probably very sensitive to even small changes in internal sugar-1-P concentration.

Concerning cytosolic UTP concentration, it was calculated at $0.23 \mathrm{mM}$ for barley endosperm (cytosolic ATP at $0.47 \mathrm{mM}$ ) (Tiessen et al., 2012) and 0.4 and $0.7 \mathrm{mM}$ for potato tubers (Farré et al., 2001) and spinach leaves (Dancer et al., 1990), respectively. Thus, the activity of UAGPase2 with GalNAc-1-P, will very much depend on even small changes of cytosolic UTP concentration ( $K_{\mathrm{m}}$ of $3 \mathrm{mM}$ ) (Supplementary Figure $\mathrm{S} 4$ ), whereas such changes will have a lesser impact on UAGPase2 activity with Glc-1-P ( $K_{\mathrm{m}}$ with UTP of $0.4 \mathrm{mM}$ ) (Supplementary Figure 55$)$. 
For UGPase and USPase, the $K_{\mathrm{m}}$ values with UTP (when Glc1-P was saturating) were 0.08-0.25 mM (Meng et al., 2008; Decker et al., 2012) and 0.08-0.19 mM (Litterer et al., 2006b; Kotake et al., 2007; Kleczkowski et al., 2011a), respectively, and thus those enzymes probably operate at saturating concentrations of UTP.

Products of the UGPase, USPase and UAGPase 2 reactions have been summarized in Figure 7. Because of high costs or commercial unavailability of the respective sugar-1-phosphates, we could not study the formation of UDP-Xyl or UDP-GlcA; however, those UDP-sugars were earlier shown to be produced by Arabidopsis USPase (see Supplementary Table S2) and are included in Figure 7. Based on this summary, it appears that the three pyrophosphorylases may decisively contribute to the synthesis of a wide range of UDP-sugars which then can be taken up as substrates by a multitude of glycosyltransferase reactions in the cell.

It seems interesting to note that plant cell wall polysaccharides, which are the most abundant components of biomass in nature (Kotake et al., 2010), are composed of at least 14 different monosaccharide moieties, most of them derived from UDP-sugars (Scheible and Pauly, 2004). As cell walls contain no GlcNAc nor GalNAc as components, it appears that among the UDP-sugar producing pyrophosphorylases it is UGPase and USPase which contribute the most to the cell wall composition. On the other hand, UAGPase may have a role in providing UDP-GlcNAc as substrate for specific glycosyltrasferases responsible for posttranslational modification of proteins with O-GlcNAc (Olszewski et al., 2010). Also, many of the $N$-linked complex glycans terminate with GlcNAc (Rayon et al., 1999), and glycoprotein $N$-glycans may contain core GlcNAc dimers (Vanholme et al., 2014), which again would require UDP-GlcNAc as substrate for their formation. The role of UDP-GalNAc is less clear since plants lack the machinery to form mucin-type (GalNAc based) glycosylations, even though there have been reports of a mucin-type glycosylations in some algal and plant species (Niemann et al., 2015). However, when the whole machinery of mucin-type glycosylation (targetpeptide, transporters and glycosyltransferase) was introduced to tobacco, the endogenous UDP-GalNAc (could originate from UDP-GlcNAc epimerase and/or UAPGase activities) was not sufficient to support GalNAc addition to the target-peptide (Daskalova et al., 2010). This suggested that the capacity for UDP-GalNAc formation in wild-type plants is low.

\section{UGPase, USPase and UAGPase2 Share Common UDP-Binding Active Site Architecture but Differ in Determinants of Sugar-Binding}

In an attempt to rationalize the observed substrate specificities, we analyzed details of substrate binding to each of the three pyrophosphorylases. To do so, we focused on aa residues which bind/ stabilize UDP-Glc, a common product of each of the three pyrophosphorylases when reacting with Glc-1-P and UTP. The analyses yielded two structural models of UDP-Glc binding for UGPase vs. USPase (Figure 6A) and for UGPase

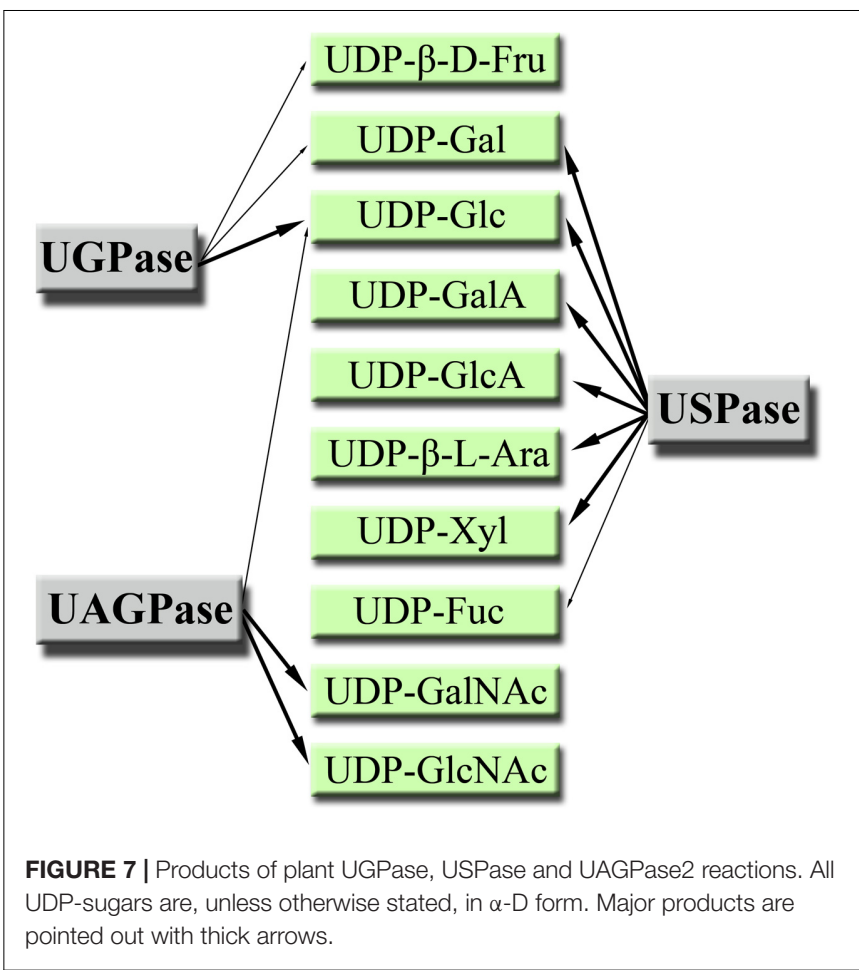

vs. UAGPase2, respectively (Figure 6B). For binding of the nucleotide portion of UDP-sugar, the models were basically identical yielding a number of conserved aa which were common for the three pyrophosphorylases. This was not surprising, given high specificity of all three pyrophosphorylases for UTP as nucleotide donor for sugar activation reaction (Figures 1-3).

On the other hand, there were significant differences in binding of a sugar portion of a given UDP-sugar, especially for aa close to hydroxyl groups at C4 and C6, and to some extent C2, of the sugar (Figure 6 and Supplementary Figure S7). Substitutions and/or configuration changes to those carbons give rise to a variety of distinct sugars which are differentially recognized by either UGPase, USPase or UAGPase2 (Figure 5). For both USPase and UAGPase2, the primary differences from UGPase must be the presence of aa interacting with groups attached to $\mathrm{C} 4$ of the sugar molecule; the two enzymes have to accept both of $\mathrm{C} 4$ epimers (Glc and Gal), whereas UGPase accepts only Glc. For UGPase, the C4 of the substrate lies in proximity of aromatic Phe292, whereas for USPase or UAGPase2 the C4 appears close to a smaller residue, such as Ile319 and Val316, respectively. Both USPase and UAGPase2 also appear to form a hydrogen bond from a Gly residues to the C4-hydroxy group of the substrate (Supplementary Figure S7). Thus it is tempting to suggest that, for UGPase, it is the presence of Phe292 and/or the lack of key glycine residues in proximity of C4 of the substrate (Gly320 and Gly317, for USPase and UAGPase2, respectively), that contributes to specificity for Glc-1-P. Interestingly, an analogous Gly308 in $L m$ USPase (which accepts GalA-1-P as substrate) is in the proximity of C4 of the sugar, while TcUSPase (homologymodeled on Leishmania USPase, PDB code 3OH4), which does not utilize GalA-1-P, has no similar residue (Yang and Bar-Peled, 
2010). Recent studies on substrate range of barley USPase (Wahl et al., 2017) revealed that it has no activity with $\alpha$-D-Fuc-1-P, and thus is distinct from Arabidopsis USPase (Supplementary Figure S3B). Further studies of these enzymes can possibly reveal residues involved in their distinct substrate specificity.

Regarding groups attached to $\mathrm{C5}$, both UGPase and UAGPase2 appear to strictly accept $-\mathrm{CH} 2 \mathrm{OH}$ at this position (Yang et al., 2010), whereas USPase tolerates a wide range of such groups $(-\mathrm{H},-\mathrm{CH} 3,-\mathrm{CH} 2 \mathrm{OH}$ and $-\mathrm{COOH})$. In this respect, UAGPase2 appears to be more related to UGPase than USPase, since both enzymes share a phenylalanine residue (Phe356 and Phe425, respectively) which is in proximity of the $-\mathrm{CH} 2 \mathrm{OH}$ attached to C5 (Figure 6 and Supplementary Figure S7). Similar to earlier studies on Arabidopsis UAGPase2 (Yang et al., 2010), the enzyme accepted only Glc-type substitutions of C6 sugar moiety, e.g., in the Yang et al. (2010) study it was not reactive with XylNAc-1-P nor GlcANAc-1-P, suggesting that it has a specific binding site for the C6 sugar group. For UAGPase2, the $N$-acetyl part of UDP-GlcNAc appears to interact with Asn250 (Supplementary Figure S7C), whereas the equivalent 3D-position in both UGPase and USPase is occupied by larger side chains of His192 and His254, respectively (Supplementary Figures S7A,B). For human UGPase, the presence of an analogous bulky histidine residue (His216) at the active site has been suggested to block GlcNAc-1-P binding (Roeben et al., 2006).

Studies on other eukaryotic UGPases and USPases, both using resolved structures (Steiner et al., 2007; Dickmanns et al., 2011) and using simulations of the reaction steps (Führing et al., 2013), have revealed that flexible portions of the enzymes are also contributing/causing the differences in substrate binding. In a study by Führing et al. (2013), the complete enzymatic cycle of Leishmania UGPase was assessed taking, e.g., into account both global (at the domain level) and local changes brought about upon substrate binding to the enzyme. Understanding the role of these conformational changes for substrate specificity of plant UDP-sugar producing pyrophosphorylases may be crucial to further rationalize/ understand how UGPase, USPase and UAGPase2 interact with their substrates and catalyze their reactions.

A common architecture at or nearby substrate-binding domains appears also to be a feature for bacterial nucleotidesugar producing pyrophosphorylases, which frequently share only less than $10 \%$ identity (based on aa sequences) with their plant counterparts (Geisler et al., 2004; Kleczkowski et al., 2004). Mild random mutagenesis of a bacterial AGPase resulted in cDNA clones coding for proteins which had their substrate specificity changed to that of UGPase and UAGPase (Sohn et al., 2006). Thus, a change of few aa could bring about a fundamental change in substrate specificity for this protein. It is unknown though whether such a "directed evolution" approach would work with plant pyrophosphorylases. On the other hand, based on comparative descriptions of the active sites of UGPase, USPase and UAGPase2 (as e.g., presented in a very simplified form in Figure 6), a more rational strategy could be devised to alter the substrate affinity of a given NDPsugar pyrophosphorylase. Obviously, for this to succeed, one would greatly benefit if crystal structures were available for all three plant UDP-sugar producing pyrophosphorylases. An open question in such analyses would also be whether such modifications do not compromise binding of activators (if any), or second substrates or catalysis. Understanding factors which determine substrate specificity of a given pyrophosphorylase may also aid future attempts to classify other nucleotide-sugar binding enzymes, such as the vast and biologically important glycosyltransferase family.

\section{UDP-Sugar Producing Pyrophosphorylases Are Affected by the Same Inhibitors}

In an earlier study (Decker et al., 2017), based on results of a chemical library screening, we have demonstrated that the activities of USPase and UGPase are inhibited by the same compounds. Subsequent hit optimization of one of the inhibitors yielded cmp \#6D, which was effective both with purified enzymes and in in vivo experiments (Decker et al., 2017). In the present study, cmp \#6D was found to inhibit also UAGPase2 activity, and the extent of inhibition was similar to that observed with UGPase and USPase (Table 1). The fact that the three enzymes are inhibited by the same compound suggests that it interacts with a site or component which is common for UGPase, USPase and UAGPase, and may lay at, or close to, the active sites of these proteins.

In addition, the Arabidopsis UAGPase2 was not affected by cmp \#41 (Table 1), which was earlier shown to inhibit UAGPase from Trypanosoma brucei (Urbaniak et al., 2013). Both barley UGPase and Arabidopsis USPase were also not affected. Based on crystal structure analyses, the Trypanosoma UAGPase 2 protein binds cmp \#41 at a unique allosteric site, which is not present in human UAGPase (Urbaniak et al., 2013). Since the structure of Arabidopsis UAGPase2 has not yet been resolved, it is unknown whether this protein contains such a site. However, in analogy to human UAGPase, the lack of inhibition by $\mathrm{cmp}$ \#41 suggests that a similar allosteric site is absent for plant UAGPase2.

The use of inhibitors to study in vivo functions of UGPase and USPase has been recently proposed (Decker et al., 2017) to overcome problems encountered by genetical approaches, where plants deficient or lacking a given pyrophosphorylase were frequently male-sterile or otherwise impaired in their reproductive abilities (e.g., Chen et al., 2007; Kotake et al., 2007; Meng et al., 2009b; Park et al., 2010; Geserick and Tenhaken, 2013a; Chen et al., 2014). Such an approach allows to use wild-type plants and the inhibitors are likely to target the same protein(s) in different plant species (Kleczkowski, 1994a; Blackwell and Zhao, 2003). Obviously, the fact that not only UGPase and USPase, but also UAGPase, are affected by the same compounds calls for more efforts to identify specific inhibitors which can discriminate between the three pyrophosphorylases. To find such a specific inhibitor, analogs of cmp \#6D and other previously identified inhibitors of UGPase and USPase (Decker et al., 2017) could be examined or, alternatively, a survey of virtual chemical libraries using in silico screening (Shoichet, 2004) could be a possibility. 


\section{AUTHOR CONTRIBUTIONS}

LK and DD conceived the original research plans; DD and LK designed all of the experiments; DD performed all of the experiments; LK and DD analyzed the data; LK wrote the article with contributions from DD.

\section{ACKNOWLEDGMENTS}

We acknowledge the help of the Protein Expertise Platform at the Department of Chemistry, Umeå University, for purification of recombinant proteins, and thank Dr. Motomitsu Kitaoka (Ibaraki, Japan) for sharing with us sugar-1-phosphates prepared

\section{REFERENCES}

Bar-Peled, M., and O’Neill, M. A. (2011). Plant nucleotide sugar formation, interconversion and salvage by sugar recycling. Annu. Rev. Plant Biol. 62, 127-155. doi: 10.1146/annurev-arplant-042110-103918

Biasini, M., Bienert, S., Waterhouse, A., Arnold, K., Studer, G., Schmidt, T., et al. (2014). SWISS-MODEL: modelling protein tertiary and quaternary structure using evolutionary information. Nucleic Acids Res. 42, 252-258. doi: 10.1093/ nar/gku340

Binet, M. R. B., Rager, M. N., and Bouvet, O. M. M. (1998). Fructose and mannose metabolism in Aeromonas hydrophila: identification of transport systems and catabolic pathways. Microbiology 144, 1113-1121. doi: 10.1099/00221287-1444-1113

Blackwell, H. E., and Zhao, Y. (2003). Chemical genetic approaches to plant biology. Plant Physiol. 133, 448-455. doi: 10.1104/pp.103.031138

Chen, R., Zhao, X., Shao, Z., Wei, Z., Wang, Y., Zhu, L., et al. (2007). Rice UDP-glucose pyrophosphorylase1 is essential for pollen callose deposition, and its cosuppression results in a new type of thermosensitive genic male sterility. Plant Cell 19, 847-861. doi: 10.1105/tpc.106.044123

Chen, Y. H., Shen, H. L., Hsu, P. J., Hwang, S. G., and Cheng, W. H. (2014). N-Acetylglucosamine-1-P uridylyltransferase 1 and 2 are required for gametogenesis and embryo development in Arabidopsis thaliana. Plant Cell Physiol. 55, 1977-1993. doi: 10.1093/pcp/pcu127

Dai, N., Petreikov, M., Portnoy, V., Katzir, N., Pharr, D. M., and Schaffer, A. A. (2006). Cloning and expression analysis of a UDP-galactose/glucose pyrophosphorylase from melon fruit provides evidence for the major metabolic pathway of galactose metabolism in raffinose oligosaccharide metabolizing plants. Plant Physiol. 142, 294-304. doi: 10.1104/pp.106.083634

Damerow, S., Lamerz, A. C., Haselhorst, T., Fuhring, J., Zarnovican, P., von Itzstein, M., et al. (2010). Leishmania UDP-sugar pyrophosphorylase: the missing link in galactose salvage? J. Biol. Chem. 285, 878-887. doi: 10.1074/jbc. M109.067223

Dancer, J., Neuhaus, H. E., and Stitt, M. (1990). Subcellular compartmentation of uridine nucleotides and nucleoside-5' -diphosphate kinase in leaves. Plant Physiol. 92, 637-641. doi: 10.1104/pp.92.3.637

Daskalova, S. M., Radder, J. E., Cichacz, Z. A., Olsen, S. H., Tsaprailis, G., Mason, H., et al. (2010). Engineering of N. benthamiana L. plants for production of $\mathrm{N}$-acetylgalactosamine-glycosylated proteins-towards development of a plant-based platform for production of protein therapeutics with mucin type O-glycosylation. BMC Biotechnol. 10:62. doi: 10.1186/1472-6750-10-62

Daudé, D., Remaud-Siméon, M., and André, I. (2012). Sucrose analogs: an attractive (bio)source for glycodiversification. Nat. Prod. Rep. 29, 945-960. doi: $10.1039 / \mathrm{c} 2 \mathrm{np} 20054 \mathrm{f}$

Decker, D., Lindberg, S., Eriksson, J., and Kleczkowski, L. A. (2014). A luminescence-based assay of UDP-sugar producing pyrophosphorylases. Anal. Methods 6, 57-61. doi: 10.1039/C3AY41811A

Decker, D., Meng, M., Gornicka, A., Hofer, A., Wilczynska, M., and Kleczkowski, L. A. (2012). Substrate kinetics and substrate effects on the quaternary in his laboratory. We also thank Dr. Matt Geisler (Southern Illinois University, Carbondale) for initiating the work on structural determinants of substrate/inhibitor binding to the pyrophosphorylases. This work was supported, in part, by the Swedish Research Council (Vetenskapsrådet) (to LK), and by Stiftelsen J. C. Kempes Minnes Stipendiefond and the Lars Hierta Memorial Foundation (to DD).

\section{SUPPLEMENTARY MATERIAL}

The Supplementary Material for this article can be found online at: http://journal.frontiersin.org/article/10.3389/fpls.2017.01610/ full\#supplementary-material

structure of barley UDP-glucose pyrophosphorylase. Phytochemistry 79, 39-45. doi: 10.1016/j.phytochem.2012.04.002

Decker, D., Öberg, C., and Kleczkowski, L. A. (2017). Identification and characterization of inhibitors of UDP-glucose and UDP-sugar pyrophosphorylases for in vivo studies. Plant J. 90, 1093-1107. doi: 10.1111/tpj.13531

Dickmanns, A., Damerow, S., Neumann, P., Schulz, E. C., Lamerz, A. C., Routier, F. H., et al. (2011). Structural basis for the broad substrate range of the UDP-sugar pyrophosphorylase from Leishmania major. J. Mol. Biol. 405, 461-478. doi: 10.1016/j.jmb.2010.10.057

Fang, W., Du, T., Raimi, O. G., Hurtado-Guerrero, R., Urbaniak, M. D., Ibrahim, A. F. M., et al. (2013). Genetic and structural validation of Aspergillus fumigatus UDP- $N$-acetylglucosamine pyrophosphorylase as an antifungal target. Mol. Microbiol. 89, 479-493. doi: 10.1111/mmi.12290

Farré, E. M., Tiessen, A., Roessner, U., Geigenberger, P., Trethewey, R. N., and Willmitzer, L. (2001). Analysis of the compartmentation of glycolytic intermediates, nucleotides, sugars, organic acids, amino acids, and sugar alcohols in potato tubers using a nonaqueous fractionation method. Plant Physiol. 127, 685-700. doi: 10.1104/pp.010280

Faust, T., Theurer, C., Eger, K., and Kreis, W. (1994). Synthesis of uridine $5^{\prime}$ - ( $\alpha$-Dfucopyranosyl diphosphate) and (digitoxigenin-3 $\beta$-yl)- $\beta$-D-fucopyranoside and enzymatic $\beta$-D-fucosylation of cardenolide aglycones in Digitalis lanata. Bioorgan. Chem. 22, 140-149. doi: 10.1006/bioo.1994.1012

Feingold, D. S. (1982). "Aldo (and keto) hexoses and uronic acids," in Encyclopedia of Plant Physiology, Vol. 13A, eds F. A. Loewus and W. Tanner (Berlin: Springer-Verlag), 3-76. doi: 10.1007/978-3-642-68275-9_1

Feingold, D. S., and Avigad, G. (1980). "Sugar nucleotide transformation in plants," in The Biochemistry of Plants, Vol. 3, eds P. K. Stumpf and E. E. Conn (New York, NY: Academic Press), 101-170.

Free, S., and Wilson, J. (1964). A mathematical contribution to structure-activity studies. J. Med. Chem. 7, 395-399. doi: 10.1021/jm00334a001

Führing, J., Cramer, J. T., Routier, F. H., Lamerz, A. C., Baruch, P., GerardySchahn, R., et al. (2013). Catalytic mechanism and allosteric regulation of UDP-glucose pyrophosphorylase from Leishmania major. ACS Catalysis 3, 2976-2985. doi: 10.1021/cs4007777

Fujii, S., Hayashi, T., and Mizuno, K. (2010). Sucrose synthase is an integral component of the cellulose synthesis machinery. Plant Cell Physiol. 51, 294-301. doi: 10.1093/pcp/pcp190

Furo, K., Nozaki, M., Murashige, H., and Sato, Y. (2015). Identification of an $\mathrm{N}$-acetylglucosamine kinase essential for UDP- $N$-acetylglucosamine salvage synthesis in Arabidopsis. FEBS Lett. 589, 3258-3262. doi: 10.1016/j.febslet.2015. 09.011

Geigenberger, P., Regierer, B., Lytovchenko, A., Leisse, A., Schauer, N., Springer, F., et al. (2004). Heterologous expression of a ketohexokinase in potato plants leads to inhibited rates of photosynthesis, severe growth retardation and abnormal leaf development. Planta 218, 569-578. doi: 10.1007/s00425-003-1152-y

Geisler, M., Wilczynska, M., Karpinski, S., and Kleczkowski, L. A. (2004). Toward a blueprint for UDP-glucose pyrophosphorylase structure/ function properties: 
homology-modeling analyses. Plant Mol. Biol. 56, 783-794. doi: 10.1007/ s11103-004-4953-x

Geserick, C., and Tenhaken, R. (2013a). UDP-sugar pyrophosphorylase is essential for arabinose and xylose recycling, and is required during vegetative and reproductive growth in Arabidopsis. Plant J. 74, 239-247. doi: 10.1111/tpj.12116

Geserick, C., and Tenhaken, R. (2013b). UDP-sugar pyrophosphorylase controls the activity of proceeding sugar-1-kinases enzymes. Plant Signal. Behav. 8:e25478. doi: $10.4161 /$ psb. 25478

Graham, D., and ap Rees, T. (1965). Occurrence of fructose-1-phosphate in tissues of higher plants. Nature 208, 88-89. doi: 10.1038/208088b0

Gupta, S. K., and Sowokinos, J. R. (2003). Physicochemical and kinetic properties of unique isozymes of UDP-glc pyrophosphorylase that are associated with resistance to sweetening in cold-stored potato tubers. J. Plant Physiol. 160, 589-600. doi: 10.1078/0176-1617-01045

Harholt, J., Suttangkakul, A., and Scheller, H. V. (2010). Biosynthesis of pectin. Plant Physiol. 153, 384-395. doi: 10.1104/pp.110.156588

Huber, S. C., and Akazawa, T. (1986). A novel sucrose synthase pathway for sucrose degradation in cultured Sycamore cells. Plant Physiol. 81, 1008-1013. doi: 10.1104/pp.81.4.1008

Kim, B. G., and Ahn, J. H. (2013). Characterization of uridine diphosphate-sugar pyrophosphorylase from Populus deltoides. J. Korean Soc. Appl. Biol. Chem. 56, 525-531. doi: 10.1007/s13765-013-3158-x

Kim, J. S., Koh, S., Shin, H., Lee, D., and Lee, S. Y. (1999). Biochemical characterization of a UDP-sugar pyrophosphorylase from Thermus caldophilus GK24. Biotechnol. Appl. Biochem. 29, 11-17.

Kimura, S., Mitsui, T., Matsuoka, T., and Igaue, I. (1992). Purification, characterization and localization of rice UDP-glucose pyrophosphorylase. Plant Physiol. Biochem. 30, 683-693.

Kleczkowski, L. A. (1994a). Inhibitors of photosynthetic enzymes/carriers and metabolism. Annu. Rev. Plant Physiol. Plant Mol. Biol. 45, 339-367. doi: 10.1146/annurev.pp.45.060194.002011

Kleczkowski, L. A. (1994b). Glucose activation and metabolism through UDP-glucose pyrophosphorylase in plants. Phytochemistry 37, 1507-1515. doi: 10.1016/S0031-9422(00)89568-0

Kleczkowski, L. A. (1996). Back to the drawing board: redefining starch synthesis in cereals. Trends Plant Sci. 1, 363-364. doi: 10.1016/1360-1385(96)83884-2

Kleczkowski, L. A., and Decker, D. (2015). Sugar activation for production of nucleotide sugars as substrates for glycosyltransferases in plants. J. Appl. Glycosci. 62, 25-36. doi: 10.5458/jag.jag.JAG-2015_003

Kleczkowski, L. A., Decker, D., and Wilczynska, M. (2011a). UDP-sugar pyrophosphorylase: a new old mechanism for sugar activation. Plant Physiol. 156, 1-8. doi: 10.1104/pp.111.174706

Kleczkowski, L. A., Geisler, M., Ciereszko, I., and Johansson, H. (2004). UDP-glucose pyrophosphorylase - an old protein with new tricks. Plant Physiol. 134, 912-918. doi: 10.1104/pp.103.036053

Kleczkowski, L. A., Geisler, M., Fitzek, E., and Wilczynska, M. (2011b). A common structural blueprint for plant UDP-sugar producing pyrophosphorylases. Biochem. J. 439, 375-379. doi: 10.1042/BJ20110730

Kleczkowski, L. A., Kunz, S., and Wilczynska, M. (2010). Mechanisms of UDP-glucose synthesis in plants. Crit. Rev. Plant Sci. 29, 191-203. doi: 10.1080/ 07352689.2010 .483578

Knop, J. K., and Hansen, R. G. (1970). Uridine diphosphate glucose pyrophosphorylase: crystallization and properties of the enzyme from human liver. J. Biol. Chem. 245, 2499-2504.

Kotake, T., Hirosawa, C., Ando, Y., and Tsumuraya, Y. (2010). Generation of nucleotide sugars for biomass formation in plants. Plant Biotechnol. 27, 231-236. doi: 10.5511/plantbiotechnology.27.231

Kotake, T., Hojo, S., Tajima, N., Matsuoka, K., Koyama, T., and Tsumuraya, Y. (2008). A bifunctional enzyme with L-fucokinase and GDP-L-fucose pyrophosphorylase activities salvages free L-fucose in Arabidopsis. J. Biol. Chem. 283, 8125-8135. doi: 10.1074/jbc.M710078200

Kotake, T., Hojo, S., Yamaguchi, D., Aohara, T., Konishi, T., and Tsumuraya, Y. (2007). Properties and physiological functions of UDP-sugar pyrophosphorylase in Arabidopsis. Biosci. Biotechnol. Biochem. 71, 761-777. doi: 10.1271/bbb.60605

Kotake, T., Yamaguchi, D., Ohzono, H., Hojo, S., Kaneko, S., Ishida, H. K., et al. (2004). UDP-sugar pyrophosphorylase with broad substrate specificity toward various monosaccharide 1-phosphates from pea sprouts. J. Biol. Chem. 279, 45728-45736. doi: 10.1074/jbc.M408716200

Lairson, L. L., Henrissat, B., Davies, G. J., and Withers, S. G. (2008). Glycosyltransferases: structures, functions and mechanisms. Annu. Rev. Biochem. 77, 521-555. doi: 10.1146/annurev.biochem.76.061005.092322

Larondelle, Y., Mertens, E., Schaftingen, E. V., and Hers, H. (1989). Fructose 2,6-bisphosphate hydrolyzing enzymes in higher plants. Plant Physiol. 90, 827-834. doi: 10.1104/pp.90.3.827

Laskowski, R. A., and Swindells, M. B. (2011). LigPlot+: multiple ligand-protein interaction diagrams for drug discovery. J. Chem. Inf. Model. 51, 2778-2786. doi: $10.1021 / \mathrm{ci} 200227 \mathrm{u}$

Litterer, L. A., Plaisance, K. L., Schnurr, J. A., Storey, K. K., Jung, H. J. G., Gronwald, J. W., et al. (2006a). Biosynthesis of UDP-glucuronic acid in developing soybean embryos: possible role of UDP-sugar pyrophosphorylase. Physiol. Plant. 128, 200-211. doi: 10.1111/j.1399-3054.2006.00728.x

Litterer, L. A., Schnurr, J. A., Plaisance, K. L., Storey, K. K., Gronwald, J. W., and Somers, D. A. (2006b). Characterization and expression of Arabidopsis UDP-sugar pyrophosphorylase. Plant Physiol. Biochem. 44, 171-180.

Liu, J., Zou, Y., Guan, W., Zhai, Y., Xue, M., Jin, L., et al. (2013). Biosynthesis of nucleotide sugars by a promiscuous UDP-sugar pyrophosphorylase from Arabidopsis thaliana (AtUSP). Bioorg. Med. Chem. Lett. 23, 3764-3768. doi: 10.1016/j.bmcl.2013.04.090

Martz, F., Wilczynska, M., and Kleczkowski, L. A. (2002). Oligomerization status, with the monomer as active species, defines catalytic efficiency of UDP-glucose pyrophosphorylase. Biochem. J. 367, 295-300. doi: 10.1042/bj20020772

McCoy, J. G., Bitto, E., Bingman, C. A., Wesenberg, G. E., Bannen, R. M., Kondrashov, D. A., et al. (2007). Structure and dynamics of UDP-glucose pyrophosphorylase from Arabidopsis thaliana with bound UDP-glucose and UTP. J. Mol. Biol. 366, 830-841. doi: 10.1016/j.jmb.2006.11.059

Meng, M., Fitzek, E., Gajowniczek, A., Wilczynska, M., and Kleczkowski, L. A. (2009a). Domain-specific determinants of catalysis/ substrate binding and the oligomerization status of barley UDP-glucose pyrophosphorylase. Biochim. Biophys. Acta 1794, 1734-1742. doi: 10.1016/j.bbapap.2009.08.009

Meng, M., Geisler, M., Johansson, H., Harholt, J., Scheller, H. V., Mellerowicz, E. J., et al. (2009b). UDP-glucose pyrophosphorylase is not rate-limiting, but is essential in Arabidopsis. Plant Cell Physiol. 50, 998-1011. doi: 10.1093/pcp/ pcp052

Meng, M., Wilczynska, M., and Kleczkowski, L. A. (2008). Molecular and kinetic characterization of two UDP-glucose pyrophosphorylases, products of distinct genes, from Arabidopsis. Biochim. Biophys. Acta 1784, 967-972. doi: 10.1016/j. bbapap.2008.02.021

Nakano, K., Omura, Y., Tagaya, M., and Fukui, T. (1989). UDP-glucose pyrophosphorylase from potato tuber: Purification and characterization. J. Biochem. 106, 528-532. doi: 10.1093/oxfordjournals.jbchem.a122886

Niemann, M. C. E., Bartrina, I., Ashikov, A., Weber, H., Novák, O., Spíchal, L., et al. (2015). Arabidopsis ROCK1 transports UDP-GlcNAc/UDP-GalNAc and regulates ER protein quality control and cytokinin activity. Proc. Natl. Acad. Sci. U.S.A. 112, 291-296. doi: 10.1073/pnas.1419050112

Ohashi, T., Cramer, N., Ishimizu, T., and Hase, S. (2006). Preparation of UDPgalacturonic acid using UDP-sugar pyrophosphorylase. Anal. Biochem. 352, 182-187. doi: 10.1016/j.ab.2006.02.026

Okazaki, Y., Shimojima, M., Sawada, Y., Toyooka, K., Narisawa, T., Mochida, K., et al. (2009). A chloroplastic UDP-glucose pyrophosphorylase from Arabidopsis is the committed enzyme for the first step of sulfolipid biosynthesis. Plant Cell 21, 892-909. doi: 10.1105/tpc.108.063925

Olszewski, N. E., West, C. M., Sassi, S. O., and Hartweck, L. M. (2010). O-GlcNAc protein modification in plants: evolution and function. Biochim. Biophys. Acta 1800, 49-56. doi: 10.1016/j.bbagen.2009.11.016

Park, J. I., Ishimizu, T., Suwabe, K., Sudo, K., Masuko, H., Hakozaki, H., et al. (2010). UDP-glucose pyrophosphorylase is rate limiting in vegetative and reproductive phases in Arabidopsis thaliana. Plant Cell Physiol. 51, 981-996. doi: $10.1093 / \mathrm{pcp} / \mathrm{pcq} 057$

Peneff, C., Ferrari, P., Charrier, V., Taburet, Y., Monnier, C., Zamboni, V., et al. (2001). Crystal structures of two human pyrophosphorylase isoforms in complexes with UDPGlc(Gal)NAc: role of the alternatively spliced insert in the enzyme oligomeric assembly and active site architecture. EMBO J. 20, 6191-6202. doi: 10.1093/emboj/20.22.6191 
Rayon, C., Cabanes-Macheteau, M., Loutelier-Bourhis, C., Salliot-Maire, I., Lemoine, J., Reiter, W. D., et al. (1999). Characterization of $N$-glycans from Arabidopsis. Application to a fucose-deficient mutant. Plant Physiol. 119, 725-734. doi: 10.1104/pp.119.2.725

Reiter, W. D. (2008). Biochemical genetics of nucleotide sugar interconversion reactions. Curr. Opin. Plant Biol. 11, 236-243. doi: 10.1016/j.pbi.2008.03.009

Ritter, J. E., Berlin, C., and Elling, L. (1996). A continuous microtiter plate assay for screening nucleotide sugar-synthesizing nucleotidyltransferases. Anal. Biochem. 234, 74-82. doi: 10.1006/abio.1996.0052

Roeben, A., Plitzko, J. M., Körner, R., Bottcher, U. M. K., Siegers, K., HayerHartl, M., et al. (2006). Structural basis for subunit assembly in UDP-glucose pyrophosphorylase from Saccharomyces cerevisiae. J. Mol. Biol. 364, 551-560. doi: 10.1016/j.jmb.2006.08.079

Sauerzapfe, B., Engels, L., and Elling, L. (2008). Broadening the biocatalytic properties of recombinant sucrose synthase1 from potato (Solanum tuberosum L.) by expression in Escherichia coli and Saccharomyces cerevisiae. Enzyme Microb. Technol. 43, 289-296. doi: 10.1016/j.enzmictec.2008.04.001

Scheible, W. R., and Pauly, M. (2004). Glycosyltransferases and cell wall biosynthesis: novel players and insights. Curr. Opin. Plant Biol. 7, 285-295. doi: $10.1016 /$ j.pbi.2004.03.006

Scheller, H. V., and Ulvskov, P. (2010). Hemicelluloses. Annu. Rev. Plant Biol. 61, 263-289. doi: 10.1146/annurev-arplant-042809-112315

Schnurr, J. A., Storey, K. K., Jung, H. J. G., Somers, D. A., and Gronwald, J. W. (2006). UDP-sugar pyrophosphorylase is essential for pollen development in Arabidopsis. Planta 224, 520-532. doi: 10.1007/s00425-006-0240-1

Sharples, S. C., and Fry, S. C. (2007). Radioisotope ratios discriminate between competing pathways of cell wall polysaccharide and RNA biosynthesis in living plant cells. Plant J. 52, 252-262. doi: 10.1111/j.1365-313X.2007.03225.x

Shoichet, B. K. (2004). Virtual screening of chemical libraries. Nature 432, 862-865. doi: $10.1038 /$ nature03197

Sohn, H., Kim, Y. S., Jin, U. H., Suh, S. J., Lee, S. C., Lee, D. S., et al. (2006). Alteration of the substrate specificity of Thermus caldophilus ADP-glucose pyrophosphorylase by random mutagenesis through error-prone polymerase chain reaction. Glycoconj. J. 23, 619-625. doi: 10.1007/s10719-006-9004-1

Steiner, T., Lamerz, A. C., Hess, P., Breithaupt, C., Krapp, S., Bourenkov, G., et al. (2007). Open and closed structures of the UDP-glucose pyrophosphorylase from Leishmania major. J. Biol. Chem. 282, 13003-13010. doi: 10.1074/jbc. M609984200

Stokes, M. J., Guther, M. L., Turnock, D. C., Prescott, A. R., Martin, K. L., Alphey, M. S., et al. (2008). The synthesis of UDP-N-acetylglucosamine is essential for bloodstream form Trypanosoma brucei in vitro and in vivo and UDP- $N$-acetylglucosamine starvation reveals a hierarchy in parasite protein glycosylation. J. Biol. Chem. 283, 16147-16161. doi: 10.1074/jbc.M709581200

Taniguchi, H., and Nakamura, M. (1972). Some properties of synthetic UDP-(2)-fructose. Agric. Biol. Chem. 36, 2185-2194.

Tiessen, A., Nerlich, A., Faix, B., Hümmer, C., Fox, S., Trafford, K., et al. (2012). Subcellular analysis of starch metabolism in developing barley seeds using a non-aqueous fractionation method. J. Exp. Bot. 63, 2071-2087. doi: 10.1093/ jxb/err408

Urbaniak, M. D., Collie, I. T., Fang, W., Aristotelous, T., Eskilsson, S., Raimi, O. G., et al. (2013). A novel allosteric inhibitor of the uridine diphosphate $N$-acetylglucosamine pyrophosphorylase from Trypanosoma brucei. ACS Chem. Biol. 8, 1981-1987. doi: 10.1021/cb400411x

Vanholme, B., Vanholme, R., Turumtay, H., Goeminne, G., Cesarino, I., Goubet, F., et al. (2014). Accumulation of N-acetylglucosamine oligomers in the plant cell wall affects plant architecture in a dose-dependent and conditional manner. Plant Physiol. 165, 290-308. doi: 10.1104/pp.113.23 3742

Wahl, C., Spiertz, M., and Elling, L. (2017). Characterization of a new UDP-sugar pyrophosphorylase from Hordeum vulgare (barley). J. Biotechnol. doi: 10.1016/ j.jbiotec.2017.03.025 [Epub ahead of print].

Yang, T., Bar-Peled, L., Gebhart, L., Lee, S. G., and Bar-Peled, M. (2009). Identification of galacturonic acid-1-phosphate kinase, a new member of the GHMP kinase superfamily in plants, and comparison with galactose-1-phosphate kinase. J. Biol. Chem. 284, 21526-21535. doi: 10.1074/jbc.M109.014761

Yang, T., and Bar-Peled, M. (2010). Identification of a novel UDP-sugar pyrophosphorylase with a broad substrate specificity in Trypanosoma cruzi. Biochem. J. 429, 533-543. doi: 10.1042/BJ20100238

Yang, T., Echols, M., Martin, A., and Bar-Peled, M. (2010). Identification and characterization of a strict and a promiscuous $\mathrm{N}$-acetylglucosamine-1-P uridylyltransferase in Arabidopsis. Biochem. J. 430, 275-284. doi: 10.1042/ BJ20100315

Yin, Y. B., Huang, J. L., Gu, X. G., Bar-Peled, M., and Xu, Y. (2011). Evolution of plant nucleotide-sugar interconversion enzymes. PLOS ONE 6:e27995. doi: 10.1371/journal.pone.0027995

Yonekura-Sakakibara, K. (2009). Functional genomics of family 1 glycosyltransferases in Arabidopsis. Plant Biotechnol. 26, 267-274. doi: $10.5511 /$ plantbiotechnology.26.267

Zhang, Q., Hrmova, M., Shirley, N. J., Lahnstein, J., and Fincher, G. B. (2006). Gene expression patterns and catalytic properties of UDP-D-glucose 4-epimerases from barley (Hordeum vulgare L.). Biochem. J. 394, 115-124. doi: 10.1042/ BJ20051329

Conflict of Interest Statement: The authors declare that the research was conducted in the absence of any commercial or financial relationships that could be construed as a potential conflict of interest.

Copyright (c) 2017 Decker and Kleczkowski. This is an open-access article distributed under the terms of the Creative Commons Attribution License (CC BY). The use, distribution or reproduction in other forums is permitted, provided the original author(s) or licensor are credited and that the original publication in this journal is cited, in accordance with accepted academic practice. No use, distribution or reproduction is permitted which does not comply with these terms. 